\title{
NONPARAMETRIC ESTIMATION OF TIME-CHANGED LÉVY MODELS UNDER HIGH-FREQUENCY DATA
}

\author{
JOSÉ E. FIGUEROA-LÓPEZ, ${ }^{*}$ Purdue University
}

\begin{abstract}
Let $\left\{Z_{t}\right\}_{t \geq 0}$ be a Lévy process with Lévy measure $v$, and let $\tau(t)=\int_{0}^{t} r(u) \mathrm{d} u$, where $\{r(t)\}_{t \geq 0}$ is a positive ergodic diffusion independent from $Z$. Based upon discrete observations of the time-changed Lévy process $X_{t}:=Z_{\tau_{t}}$ during a time interval $[0, T]$, we study the asymptotic properties of certain estimators of the parameters $\beta(\varphi):=\int \varphi(x) v(\mathrm{~d} x)$, which in turn are well known to be the building blocks of several nonparametric methods such as sieve-based estimation and kernel estimation. Under uniform boundedness of the second moments of $r$ and conditions on $\varphi$ necessary for the standard short-term ergodic property $\lim _{t \rightarrow 0} \mathrm{E} \varphi\left(Z_{t}\right) / t=\beta(\varphi)$ to hold, consistency and asymptotic normality of the proposed estimators are ensured when the time horizon $T$ increases in such a way that the sampling frequency is high enough relative to $T$.
\end{abstract}

Keywords: Lévy process; nonparametric estimation; high-frequency-based inference; stochastic volatility

2000 Mathematics Subject Classification: Primary 60J75; 60F05; 62M05

\section{Introduction}

Historically, Brownian motion has been the model of choice to describe the evolution of a random measurement whose value is the result of a large number of small shots occurring through time with high frequency. This is indeed the situation with stock prices whose value is the result of a high number of agents posting bids and asking prices almost at all times. However, processes exhibiting infinitely many jumps in any finite time horizon $[0, T]$ are arguably better approximations to such high-activity stochastic processes. A Lévy process is a natural extension of Brownian motion which preserves the tractable statistical properties of its increments, but relaxes the continuity of paths. The previous considerations motivated an explosion of financial-price models driven by Lévy processes with infinite jump activity. The simplest of these models postulates that the price of a commodity (say a stock) at time $t$ is determined by

$$
S_{t}:=S_{0} \mathrm{e}^{X_{t}}
$$

where $X:=\left\{X_{t}\right\}_{t \geq 0}$ is a Lévy process. Even this simple extension of the classical BlackScholes model, in which $X$ is simply a Brownian motion with drift, is able to accommodate several features commonly observed in the returns of financial assets, such as heavy tails, highkurtosis, and asymmetry. Among the better known models are the variance Gamma model of [19], the CGMY model of [5], and the generalized hyperbolic motion of [1] and [9] (see also [2] and [8]).

Received 7 January 2009; revision received 15 July 2009.

* Postal address: Department of Statistics, Purdue University, West Lafayette, IN 47907-2066, USA.

Email address: figueroa@stat.purdue.edu 
Even though the geometric Lévy paradigm (1.1) incorporates several desirable stylized features, the model has several shortcomings, especially in accounting for the so-called volatility clustering and leverage phenomena exhibited by real financial data. Roughly speaking, the former effect refers to the fact that there are periods of high variability in the market, followed by periods of low variability. People usually say that 'high-volatility' events tend to cluster in time. Leverage refers to the empirical observation that returns seem to be negatively correlated with volatility. These two effects cannot be captured by model (1.1). To explain why this is the case and to motivate the use of random clocks, let us study the realized variation up to time $t$ of the log returns in the periods $\left[t_{0}, t_{1}\right], \ldots,\left[t_{n-1}, t_{n}\right]$ :

$$
V_{\pi}(t):=\sum_{\left\{i: t_{i} \leq t\right\}} \log ^{2}\left(\frac{S_{t_{i}}}{S_{t_{i-1}}}\right),
$$

where $\pi: t_{0}=0<t_{1}<\cdots<t_{n}:=T<\infty$. When the mesh $\bar{\pi}:=\max _{i}\left\{t_{i}-t_{i-1}\right\}$ of the partition is small, we can think of the increment $V_{\pi}(t)-V_{\pi}(s)$ as a measure of the volatility of the stock during the period $[s, t]$. Under model (1.1),

$$
V_{\pi}(t)=\sum_{\left\{i: t_{i} \leq t\right\}}\left(X_{t_{i}}-X_{t_{i-1}}\right)^{2},
$$

which is well known to converge, in probability, to the quadratic variation of the process,

$$
[X]_{t}:=\sigma^{2} t+\sum_{s \leq t} \Delta X_{s}
$$

as $\bar{\pi} \rightarrow 0$. In this case, the realized variations in consecutive time periods of equal time length $\Delta$, say $V_{\pi}(\Delta), V_{\pi}(2 \Delta)-V_{\pi}(\Delta)$, etc., will look like white noise (i.e. independent, identically distributed random variables) and will not exhibit the volatility clustering phenomenon.

In recent years subordinated Lévy processes have been proposed to incorporate the intermittency and leverage phenomena (cf. [4] and [6]). Concretely, these models postulate that the asset price at time $t$ is given by (1.1) with

$$
X_{t}:=Z_{\tau(t)}
$$

where $Z$ is a Lévy process and $\{\tau(t)\}_{t \geq 0}$ stands for a nondecreasing absolutely continuous process. This approach leads to a geometric time-changed Lévy model:

$$
S_{t}=S_{0} \mathrm{e}^{Z_{\tau(t)}},
$$

where the process $\tau$ plays the role of a 'business' clock which may reflect nonsynchronous trading effects or a 'cumulative measure of economic activity'. To incorporate volatility clustering, random clocks $\{\tau(t)\}_{t \geq 0}$ of the form

$$
\tau(t):=\int_{0}^{t} r(u) \mathrm{d} u,
$$

with $\{r(t)\}_{t \geq 0}$ being a positive mean-reverting process, are plausible choices. This crucial observation was first noticed by Carr et al. [6], who specialized further their model to consider particular parametric models for $Z$ (such as normal inverse Gaussian or variance gamma processes) and explicit positive ergodic diffusions for $r$ such as the Cox-Ingersoll-Ross (CIR) 
process. Roughly speaking, the rate process $r$ controls the volatility of the process; for instance, in time periods where $r$ is high the 'business time' $\tau$ runs faster, resulting in more frequent jump times. More formally, under model (1.1) with $X$ as in (1.3) and assuming that $\tau$ is independent of the Lévy process $Z$, the realized variation (1.2) of the log returns converges to

$$
\sigma^{2} \tau(t)+\sum_{s \leq \tau(t)} \Delta Z_{s},
$$

where $\sigma$ is the variance of the Brownian component of $Z$. The observable volatility during a time period $[t, u]$ will be given by

$$
\sigma^{2}\{\tau(u)-\tau(t)\}+\sum_{\tau(t)<s \leq \tau(u)} \Delta Z_{s} .
$$

Thus, under (1.4) with a mean-reverting process $\{r(t)\}_{t \geq 0}$, there will be periods $[t, u]$ of high volatility (which correspond to periods where the process $r$ takes on a high level) and periods $[t, u]$ of low volatility (which correspond to periods where $r$ takes on a low level).

Time-changed Lévy processes are one step further in the trend of increasingly complex models that are aimed at incorporating the so-called stylized features of asset prices. Considerably less effort has been devoted to analyzing the potential departures from the presumed model. One recent approach to deal with the latter issue is the adoption of general nonparametric models for the functional parameters of the underlying process, hence reducing the estimation bias resulting from assuming an inadequate parametric model. In the case of a Lévy model $Z$, this parameter could be the so-called Lévy density $s(\cdot)$, which dictates the jump dynamics of the process and is the main object of interest in the present paper. The value of $s$ at a point $x_{0}$ determines how frequently jumps of size close to $x_{0}$ occur per unit time. Concretely, the function $s$ is such that

$$
\int_{A} s(x) \mathrm{d} x=\frac{1}{t} \mathrm{E}\left[\sum_{s \leq t} \chi_{A}\left(\Delta Z_{S}\right)\right]
$$

for any Borel set $A$ and $t>0$. Here, $\Delta Z_{t} \equiv Z_{t}-Z_{t^{-}}$denotes the magnitude of the jump of $Z$ at time $t$, and $\chi_{A}(x)=1$ if $x \in A$ and 0 otherwise. Thus,

$$
v(A):=\int_{A} s(x) \mathrm{d} x,
$$

called the Lévy measure of the process, is the average number of jumps (per unit time) whose magnitudes fall in the set $A$. For instance, if $v((0, \infty))=0$ then $Z$ will exhibit only jumps of negative size. In the context of financial applications, an empirical assessment of the possible sudden price shifts of the underlying assets is critical as these shifts play a key role in developing appropriate risk management and investment strategies.

The challenge of devising nonparametric methods for the Lévy density $s$ of $Z$ lies in the fact that the jumps are latent (unobservable) variables, since in practice only discrete observations of the process are available. It is natural to devise statistical methodologies based on highfrequency observations since this type of data will contain more relevant information about the jumps of the process and, hence, about the Lévy density $s$. Such a high-frequency-based statistical approach has played a central role in the recent literature on nonparametric estimation for Lévy processes (see, e.g. [10], [13], [20], [24], and [25]). For instance, under discrete 
observations of a pure Lévy process $X$ at times $\pi: 0=t_{0}<\cdots<t_{n}=T$, Woerner [24], and also independently Figueroa-López [10], proposed the estimators

$$
\hat{\beta}^{\pi}(\varphi):=\frac{1}{t_{n}} \sum_{k=1}^{n} \varphi\left(X_{t_{k}}-X_{t_{k-1}}\right)
$$

as consistent estimators for the integral parameter

$$
\beta(\varphi):=\int \varphi(x) s(x) \mathrm{d} x,
$$

where $\varphi$ is a given 'test function'. We can think of statistic (1.5) as the realized $\varphi$-variation of the process $X$ per unit time based on the sampling observations $X_{t_{0}}, \ldots, X_{t_{n}}$. In [10], the proposed estimators were used to devise nonparametric estimators $\hat{s}$ for $s$ via Grenander's method of sieves. The problem of model selection was analyzed further in [13] and [15], where it was proved that sieve estimators $\tilde{s}_{T}$ can match the rate of convergence of the minimax risk of estimators $\hat{s}$. Concretely, it turns out that

$$
\limsup _{T \rightarrow \infty} \frac{\mathrm{E}\left\|s-\tilde{s}_{T}\right\|^{2}}{\inf _{\hat{s}} \sup _{s \in \Theta} \mathrm{E}\|s-\hat{s}\|^{2}}<\infty,
$$

where $[0, T]$ is the time horizon over which we observe the process $X, \Theta$ is a certain class of smooth functions, and the infimum in the denominator is over all estimators $\hat{s}$ which are based on the whole trajectory $\left\{X_{t}\right\}_{t \leq T}$. The optimal rate of the estimator $\tilde{s}_{T}$ is attained by appropriately choosing the dimension of the sieve and the sampling frequency as a function of $T$ and the smoothness of the class of functions $\Theta$. In [11], the sieve estimators of [13] were also used to build confidence intervals and confidence bands for the Lévy density $s$.

In this paper we consider the problem of making statistical inferences for model (1.3) when we have at hand high-frequency sampling observations of $X$. A recent treatment of the problem of predicting (estimating) the business clock process $\tau(t):=\int_{0}^{t} r(u) \mathrm{d} u$ was given in [25]. We concentrate here on estimating the Lévy density $s$ of the Lévy process $Z$. A natural question is the following: how does the random time $\tau$ affect the statistical properties of estimator (1.5)? We prove that when the rate process $r$ in (1.4) is a positive ergodic diffusion independent of the Lévy process $Z,(1.5)$ is still a consistent estimator for (1.6) up to a constant, provided that the time horizon $T$ and sampling frequency converge to infinity at suitable rates. Roughly speaking, suppose that the following conditions hold true.

(i) $\varphi$ is a continuous, locally bounded function such that

$$
\sup _{t>0} \frac{1}{t} \mathrm{E}\left|\varphi\left(Z_{t}\right)\right|<\infty,
$$

and $\varphi(x) \rightarrow 0$ as $x \rightarrow 0$ at an 'appropriate rate' (see Condition 2.1, below).

(ii) $\{r(t)\}_{t \geq 0}$ is an ergodic positive solution of the stochastic differential equation

$$
\mathrm{d} r(t)=b(r(t)) \mathrm{d} t+\sigma(r(t)) \mathrm{d} W_{t},
$$

such that $r$ is independent of $Z$ and

$$
\sup _{t \geq 0} \mathrm{E} r^{2}(t)<\infty
$$


(iii) The time horizon $T$ and the sampling times $\pi_{T}$ are such that $T \rightarrow \infty$ and $T \bar{\delta}_{T}^{2} \rightarrow 0$ as $T \rightarrow \infty$, where $\bar{\delta}_{T}$ is the largest time span between consecutive observations.

Then it follows that, as $T \rightarrow \infty$,

$$
\hat{\beta}^{\pi_{T}}(\varphi) \stackrel{\mathrm{P}}{\rightarrow} \bar{\zeta} \beta(\varphi), \quad \mathrm{E} \hat{\beta}^{\pi_{T}}(\varphi) \rightarrow \bar{\zeta} \beta(\varphi)
$$

where $\bar{\zeta}:=\bar{\zeta}(r)$ is the expectation of the invariant distribution of $r$. Furthermore, under the stronger assumption that

$$
\sup _{t \geq 0} \mathrm{E}|r(t)|^{2+\varepsilon}<\infty
$$

for some $\varepsilon>0$, the condition $T \bar{\delta}_{T}^{2} \rightarrow 0$ as $T \rightarrow \infty$ is not needed for (1.9).

By an ergodic diffusion we mean a strong continuous Markov process $\{r(t)\}_{t \geq 0}$ that takes values on an interval $I:=(a, b) \subset \mathbb{R}$, and that is regular and recurrent (see, e.g. [17, pp. 376]). Such a process admits a unique invariant probability measure $\zeta$, which in turn satisfies the ergodic property

$$
\lim _{t \rightarrow \infty} \frac{1}{t} \int_{0}^{t} g(r(u)) \mathrm{d} u=\int_{I} g(x) \zeta(\mathrm{d} x) \quad \text { almost surely (a.s.) }
$$

for any $g \in \mathbb{L}^{1}(\zeta)$ (cf. [17, Theorem 20.14]). A model that meets condition (ii) and that is a typical choice in applications (cf. [6]) is the CIR process

$$
\mathrm{d} r(t)=\alpha(m-r(t)) \mathrm{d} t+v \sqrt{r(t)} \mathrm{d} W_{t},
$$

with positive $\alpha, v$, and $m$ such that $\alpha m / v^{2}>\frac{1}{2}$. It will turn out that the consistency (1.9) is a consequence of the ergodic property (1.11) and the consistency of the estimator (1.5) when the underlying process $X$ is a pure Lévy process.

Let us remark that the independence assumption between $Z$ and $r$ is a drawback from a financial point of view. We could think of ad hoc treatments to incorporate certain degrees of dependence, such as common driving factors for $r$ and $Z$, but we will not explore this direction in this work. Note also that without loss of generality we can assume that $\bar{\zeta}(r):=1$ since, for an arbitrary Lévy process $Z$ and a diffusion $r$, we can write the time-changed Lévy process (1.3)-(1.4) as follows:

$$
Z_{\tau(t)}=\hat{Z}_{\hat{\tau}(t)},
$$

with $\hat{Z}_{t}:=Z_{\bar{\zeta}(r) t}, \hat{\tau}(t):=\int_{0}^{t} \hat{r}(u) \mathrm{d} u$, and $\hat{r}(t):=r(t) / \bar{\zeta}(r)$. The process $\hat{Z}$ is again a Lévy process satisfying (1.7) with Lévy density $\bar{\zeta}(r) s$. Similarly, $\hat{r}$ is a positive ergodic solution of the stochastic differential equation

$$
\mathrm{d} \hat{r}(t)=\hat{b}(\hat{r}(t)) \mathrm{d} t+\hat{\sigma}(\hat{r}(t)) \mathrm{d} W_{t},
$$

with $\hat{b}(x):=\bar{\zeta}(r)^{-1} b(\bar{\zeta}(r) x)$ and $\hat{\sigma}(x):=\bar{\zeta}(r)^{-1} \sigma(\bar{\zeta}(r) x)$. Clearly, $\hat{r}$ satisfies (1.8) and $\bar{\zeta}(\hat{r})=1$.

In the last part of the paper we obtain a central limit theorem for the estimators $\hat{\beta}^{\pi_{T}}(\varphi)$ in (1.5) with scaling constant $T^{1 / 2}$ and centering constants

$$
\check{\beta}^{\pi_{T}}(\varphi):=\frac{1}{t_{n}} \sum_{k=1}^{n} \mathrm{E}\left[\varphi\left(X_{t_{k}}-X_{t_{k-1}}\right) \mid \mathcal{F}_{t_{k-1}}^{X, \tau}\right],
$$

where $\mathcal{F}_{t}^{X, \tau}=\sigma\left(X_{u}: u \leq t\right) \vee \sigma(\tau(u): u \leq t)$. Concretely, using central limit theorems for 
martingale differences (see, e.g. [3, Section 18]), we show that

$$
T^{1 / 2}\left(\hat{\beta}^{\pi_{T}}(\varphi)-\check{\beta}^{\pi_{T}}(\varphi)\right) \stackrel{\mathrm{D}}{\rightarrow} \sigma(\varphi) \mathcal{N}(0,1)
$$

as $T \rightarrow \infty$ and $\bar{\delta}_{T} \rightarrow 0$, with $\sigma^{2}(\varphi):=\bar{\zeta} \beta\left(\varphi^{2}\right)$. Under certain conditions, the statistics $\check{\beta}^{\pi_{T}}(\varphi)$ themselves satisfy the central limit theorem

$$
T^{1 / 2}\left(\check{\beta}^{\pi_{T}}(\varphi)-\bar{\zeta} \beta(\varphi)\right) \stackrel{\mathrm{D}}{\rightarrow} \beta(\varphi) \Gamma^{1 / 2} \mathcal{N}(0,1)
$$

whenever $T \bar{\delta}_{T} \rightarrow 0$ as $n \rightarrow \infty$, for a certain positive constant $\Gamma$ depending on the process $r$. Such a result suggests a central limit theorem of the form

$$
T^{1 / 2}\left(\hat{\beta}^{\pi_{T}}(\varphi)-\bar{\zeta} \beta(\varphi)\right) \stackrel{\mathrm{D}}{\rightarrow}\left(\sigma^{2}(\varphi)+\beta^{2}(\varphi) \Gamma\right)^{1 / 2} \mathcal{N}(0,1) ;
$$

however, the latter limit is still under investigation and will be addressed in a future work.

The paper is structured as follows. In Section 2 we show the consistency for time-changed Lévy models with a general random clock $\tau$. We propose the limit

$$
\lim _{n \rightarrow \infty} \mathrm{E}\left[\frac{1}{\tau\left(t_{n}^{n}\right)} \sum_{k=1}^{n}\left(\Delta_{k}^{n} \tau\right) \mathbf{1}_{\left\{\Delta_{k}^{n} \tau \geq t_{0}\right\}}\right]=0
$$

for arbitrary $t_{0}>0$, where $\Delta_{k}^{n} \tau:=\tau\left(t_{k}^{n}\right)-\tau\left(t_{k-1}^{n}\right)$, as a key assumption on the random clock $\tau$ for consistency to hold. As an application, the case of a pure Lévy model is considered, extending a former result by Woerner [24] to nonregular sampling schemes and simpler functions $\varphi$. In Section 3 we proceed to investigate conditions under which (1.12) holds when $\tau$ is driven by a positive ergodic diffusion $\{r(t)\}_{t \geq 0}$ via (1.4). The case of general sampling schemes is discussed in Section 4. In particular, it is proved that, under (1.10), the rate condition $T \bar{\delta}_{T}^{2} \rightarrow 0$ is not needed for consistency. Finally, the asymptotic normality of the estimators is addressed in Section 5.

\section{Estimation of integrals of the Lévy measure}

We consider a time-changed Lévy model of the form (1.3), where $\left\{Z_{t}\right\}_{t>0}$ is a Lévy process with generating triplet $\left(b, \sigma^{2}, v\right)$ and $\{\tau(t)\}_{t \geq 0}$ is as in (1.4) for a general nonnegative process $r$ that is independent of $Z$. Suppose that we sample the process $X$ over a finite time horizon $\left[0, T_{n}\right]$ at discrete times $0=t_{0}^{n}<\cdots<t_{n}^{n}=T_{n}$. In this section we provide conditions for the convergence in probability of the realized $\varphi$-variations

$$
\hat{\beta}_{n}(\varphi):=\frac{1}{t_{n}^{n}} \sum_{k=1}^{n} \varphi\left(X_{t_{k}^{n}}-X_{t_{k-1}^{n}}\right)
$$

as the time-horizon $T_{n}$ tends to $\infty$ and the largest time span between observations, $\bar{\delta}^{n}:=$ $\max _{k}\left\{t_{k+1}^{n}-t_{k}^{n}\right\}$, tends to 0 . In the case of a pure Lévy process (namely, $\tau(t)=t$ and $X=Z$ ) and equally spaced time points, Woerner [24] (see Theorems 4.2 and 5.1 therein) considered this problem under some additional regularity conditions that can be greatly simplified, as will be shown here (see Theorem 2.2, below).

In order to study the behavior of (2.1), we first survey the asymptotics of the following statistics:

$$
\tilde{\beta}_{n}(\varphi):=\frac{1}{\tau\left(t_{n}^{n}\right)} \sum_{k=1}^{n} \varphi\left(Z_{\tau\left(t_{k}^{n}\right)}-Z_{\tau\left(t_{k-1}^{n}\right)}\right),
$$


when the time horizon $T_{n}=t_{n}^{n} \rightarrow \infty$ and $\bar{\delta}^{n} \rightarrow 0$. Throughout this section, we shall write $\tau_{k}^{n}:=\tau\left(t_{k}^{n}\right)$. Note that, owing to the independence of $Z$ and $\tau$, the convergence in probability of $\tilde{\beta}_{n}(\varphi)$ will follow from the pure Lévy case $(Z=X)$ if $\tau_{n}^{n} \rightarrow \infty$ a.s. as $n \rightarrow \infty$, and

$$
\max _{k}\left(\tau_{k}^{n}-\tau_{k-1}^{n}\right) \rightarrow 0 \quad \text { a.s. as } n \rightarrow \infty
$$

However, condition (2.3) is rather unsatisfactory as it translates into assuming that almost all paths $t \rightarrow \tau(t)$ are uniformly continuous in all $\mathbb{R}_{+}$since the time horizon $t_{n}^{n}$ is increasing.

We first review a crucial preliminary result. It is well known that

$$
\lim _{t \rightarrow 0} \frac{1}{t} \operatorname{E} \varphi\left(X_{t}\right)=\int \varphi(x) v(\mathrm{~d} x)
$$

for any bounded $v$-continuous function $\varphi$ vanishing in a neighborhood of the origin (cf. [21, Corollary 8.9]). Consider the following class of locally bounded (but potentially unbounded) functions:

$$
\begin{array}{r}
f(v):=\left\{g: \mathbb{R} \rightarrow \mathbb{R}_{+}: \int_{|x|>1} g(x) v(\mathrm{~d} x)<\infty, g(x)=p(x) q(x),\right. \\
\text { where } p \text { is subadditive and } q \text { is submultiplicative }\} .
\end{array}
$$

Building on results in [16] and [24], Figueroa-López [12] proved that the limit

$$
\breve{\beta}(\varphi):=\lim _{t \rightarrow 0} \frac{1}{t} \mathrm{E} \varphi\left(X_{t}\right)
$$

exists provided that the following conditions hold.

Conditions 2.1. (i) $\varphi$ is v-continuous and locally bounded.

(ii) There exists a function $g$ in $\&(v)$ such that

$$
\limsup _{|x| \rightarrow \infty} \frac{|\varphi(x)|}{g(x)}<\infty
$$

(iii) $\varphi(x) \rightarrow 0$ as $x \rightarrow 0$ under any of the following conditions:

(a) $\varphi(x)=o\left(|x|^{2}\right)$;

(b) $\varphi(x)=O\left(|x|^{r}\right)$ for some $r \in(1,2)$ such that $\int\left(|x|^{r} \wedge 1\right) \nu(\mathrm{d} x)<\infty$ and $\sigma=0$;

(c) $\varphi(x)=o(|x|), \int(|x| \wedge 1) v(\mathrm{~d} x)<\infty$, and $\sigma=0$;

(d) $\varphi(x)=O\left(|x|^{r}\right)$ for some $r \in(0,1)$ such that $\int\left(|x|^{r} \wedge 1\right) v(\mathrm{~d} x)<\infty, \sigma=0$, and $\bar{b}:=b-\int_{|x| \leq 1} x \vee(\mathrm{d} x)=0 ;$

(e) $\varphi(x) \sim x^{2}$;

(f) $\varphi(x) \sim|x|$ and $\sigma=0$. 
Moreover, (2.6) is given as follows, depending on which condition in Conditions 2.1(iii) is satisfied:

$$
\breve{\beta}(\varphi):= \begin{cases}\beta(\varphi) & \text { if any (a)-(d) is true, } \\ \sigma^{2}+\beta(\varphi) & \text { if (e) is true, } \\ |\bar{b}|+\beta(\varphi) & \text { if (f) is true, }\end{cases}
$$

where, as before, $\beta(\varphi):=\int \varphi(x) v(x) \mathrm{d} x$. Note that Conditions 2.1 imply that $\beta(|\varphi|)<\infty$.

We are ready to study the asymptotic behavior of (2.2). The following result gives conditions for asymptotic unbiasedness.

Proposition 2.1. Assume that the following statements hold:

(i) $\varphi$ is a continuous function satisfying Conditions 2.1 and

$$
M_{\varphi}:=\sup _{t>0} \frac{1}{t} \mathrm{E}\left|\varphi\left(Z_{t}\right)\right|<\infty
$$

(ii) the process $\{\tau(t)\}_{t \geq 0}$ is nondecreasing and càdlàg (right continuous with left limits), independent of $Z$, such that $\tau(t)>0$ a.s. for any $t>0$, and

$$
\lim _{n \rightarrow \infty} \mathrm{E}\left[\frac{1}{\tau\left(t_{n}^{n}\right)} \sum_{k=1}^{n}\left(\Delta_{k}^{n} \tau\right) \mathbf{1}_{\left\{\Delta_{k}^{n} \tau \geq t_{0}\right\}}\right]=0
$$

for any $t_{0}>0$, where $\Delta_{k}^{n} \tau:=\tau\left(t_{k}^{n}\right)-\tau\left(t_{k-1}^{n}\right)$.

Then, the statistics $\tilde{\beta}_{n}(\varphi)$ in (2.2) are asymptotically unbiased estimators for the parameter $\breve{\beta}(\varphi)$ in (2.7) as $n \rightarrow \infty$.

Proof. Conditioning on $\left\{\tau_{k}^{n}\right\}_{k \leq n}$ and using the independence between $\tau$ and $X$ (see Appendix B for more details), we obtain

$$
\mathrm{E} \tilde{\beta}_{n}(\varphi)=\mathrm{E} \frac{1}{\tau_{n}^{n}} \sum_{k=1}^{n} H_{\varphi}\left(\Delta_{k}^{n} \tau\right)
$$

where $H_{\varphi}(t):=\mathrm{E} \varphi\left(Z_{t}\right)$. Then,

$$
\left|\mathrm{E} \tilde{\beta}_{n}(\varphi)-\breve{\beta}(\varphi)\right| \leq \mathrm{E} \frac{1}{\tau_{n}^{n}} \sum_{k=1}^{n}\left|\frac{H_{\varphi}\left(\Delta_{k}^{n} \tau\right)}{\Delta_{k}^{n} \tau}-\breve{\beta}(\varphi)\right| \Delta_{k}^{n} \tau,
$$

under the convention that $0 / 0=0$. For $\varepsilon>0$, let $t_{0}:=t_{0}(\varepsilon)>0$ such that if $t<t_{0}$ then $\left|H_{\varphi}(t) / t-\breve{\beta}(\varphi)\right|<\varepsilon$. Then, breaking up the above summation into the $k$ s such that $\Delta_{k}^{n} \tau<t_{0}$ and its complement, and using the fact that $\sum_{k} \Delta_{k}^{n} \tau=\tau_{n}^{n}$, we obtain

$$
\left|\mathrm{E} \tilde{\beta}_{n}(\varphi)-\breve{\beta}(\varphi)\right| \leq \varepsilon+\left(M_{\varphi}+|\breve{\beta}(\varphi)|\right) \mathrm{E} \frac{1}{\tau_{n}^{n}} \sum_{k=1}^{n} \Delta_{k}^{n} \tau \mathbf{1}_{\left\{\Delta_{k}^{n} \tau \geq t_{0}\right\}} \rightarrow \varepsilon \quad \text { as } n \rightarrow \infty .
$$

This proves the result since $\varepsilon$ is arbitrary.

We proceed to show that the conditions above are also sufficient for the consistency of estimator (2.2). We first need to introduce a truncated version of (2.2) via the following lemma. 
Lemma 2.1. Let

$$
\tilde{\beta}_{n}^{t}(\varphi):=\frac{1}{\tau_{n}^{n}} \sum_{k=1}^{n} \varphi\left(Z_{\tau_{k}^{n}}-Z_{\tau_{k-1}^{n}}\right) \mathbf{1}_{\left\{\left|\varphi\left(Z_{\tau_{k}^{n}}-Z_{\tau_{k-1}^{n}}\right)\right| \leq \tau_{n}^{n}\right\}},
$$

and assume that Proposition 2.1(i)-(ii) hold as well as

(i) $\tau_{n}^{n} \rightarrow \infty$ a.s.

Then,

$$
\lim _{n \rightarrow \infty} \mathrm{E}\left[\tilde{\beta}_{n}^{t}(\varphi)-\tilde{\beta}_{n}(\varphi)\right]=0
$$

Proof. For a given $T_{0}>0$, let $t_{0}:=t_{0}\left(T_{0}\right)$ be such that if $0<t<t_{0}$ then

$$
\mathrm{E}\left[\left|\varphi\left(Z_{t}\right)\right| \mathbf{1}_{\left\{\left|\varphi\left(Z_{t}\right)\right|>T_{0}\right\}}\right] \leq 2 t\left(\int|\varphi(x)| \mathbf{1}_{\left\{|\varphi(x)|>T_{0}\right\}} v(\mathrm{~d} x) \vee T_{0}^{-1}\right) .
$$

Such a $t_{0}>0$ exists since $|\varphi(\cdot)| \mathbf{1}_{\left\{|\varphi(\cdot)|>T_{0}\right\}}$ satisfies Conditions 2.1 with behavior (iii)(a) and, thus,

$$
\lim _{t \rightarrow 0} \frac{1}{t} \mathrm{E}\left[\left|\varphi\left(Z_{t}\right)\right| \mathbf{1}_{\left\{\left|\varphi\left(Z_{t}\right)\right|>T_{0}\right\}}\right]=\int|\varphi(x)| \mathbf{1}_{\left\{|\varphi(x)|>T_{0}\right\}} v(\mathrm{~d} x) .
$$

Note that

$$
\begin{aligned}
\mathrm{E}\left|\tilde{\beta}_{n}^{t}(\varphi)-\tilde{\beta}_{n}(\varphi)\right| \leq & \mathrm{E} \frac{1}{\tau_{n}^{n}} \sum_{k=1}^{n}\left|\varphi\left(Z_{\tau_{k}^{n}}-Z_{\tau_{k-1}^{n}}\right)\right| \mathbf{1}_{\left\{\left|\varphi\left(Z_{\tau_{k}^{n}}-Z_{\tau_{k-1}^{n}}\right)\right|>\tau_{n}^{n}\right\}} \\
\leq & \mathrm{E} \frac{1}{\tau_{n}^{n}} \sum_{k=1}^{n}\left|\varphi\left(Z_{\tau_{k}^{n}}-Z_{\tau_{k-1}^{n}}\right)\right| \mathbf{1}_{\left\{\tau_{n}^{n} \leq T_{0}\right\}} \\
& +\mathrm{E} \frac{1}{\tau_{n}^{n}} \sum_{k=1}^{n}\left|\varphi\left(Z_{\tau_{k}^{n}}-Z_{\tau_{k-1}^{n}}\right)\right| \mathbf{1}_{\left\{\left|\varphi\left(Z_{\tau_{k}^{n}}-Z_{\tau_{k-1}^{n}}\right)\right|>T_{0}\right\}} .
\end{aligned}
$$

Conditioning on $\left\{\tau_{k}^{n}\right\}_{k \leq n}$ in the last two expectations and using the stationary increments of $Z$, it is evident that $\mathrm{E}\left|\tilde{\beta}_{n}^{t}(\varphi)-\tilde{\beta}_{n}(\varphi)\right|$ can be bounded by

$$
\mathrm{E} \frac{1}{\tau_{n}^{n}} \sum_{k=1}^{n} H_{|\varphi|}\left(\Delta_{k}^{n} \tau\right) \mathbf{1}_{\left\{\tau_{n}^{n} \leq T_{0}\right\}}+\mathrm{E} \frac{1}{\tau_{n}^{n}} \sum_{k=1}^{n} H_{|\varphi| \mathbf{1}_{\left\{|\varphi|>T_{0}\right\}}}\left(\Delta_{k}^{n} \tau\right),
$$

where, as before, $H_{\varphi}(t):=\mathrm{E} \varphi\left(Z_{t}\right)$. Using the facts that $M_{\varphi}:=\sup _{t>0}(1 / t) \mathrm{E}\left|\varphi\left(Z_{t}\right)\right|<\infty$ and $\sum_{k=1}^{n} \tau_{k}^{n}=\tau_{n}^{n}$, the first term in (2.15) is bounded by $M_{\varphi} \mathrm{P}\left(\tau_{n}^{n} \leq T_{0}\right)$. Similarly, using the same identities and (2.13), the second term can be bounded as follows:

$$
\begin{aligned}
& \mathrm{E} \frac{1}{\tau_{n}^{n}} \sum_{k=1}^{n} H_{|\varphi| \mathbf{1}_{\left\{|\varphi|>T_{0}\right\}}}\left(\Delta_{k}^{n} \tau\right)\left\{\mathbf{1}_{\left\{\Delta_{k}^{n} \tau<t_{0}\right\}}+\mathbf{1}_{\left\{\Delta_{k}^{n} \tau \geq t_{0}\right\}}\right\} \\
& \quad \leq 2\left(\int|\varphi(x)| \mathbf{1}_{\left\{|\varphi(x)|>T_{0}\right\}} v(\mathrm{~d} x) \vee T_{0}^{-1}\right)+M_{\varphi} \mathrm{E}\left[\frac{1}{\tau\left(t_{n}^{n}\right)} \sum_{k=1}^{n}\left(\Delta_{k}^{n} \tau\right) \mathbf{1}_{\left\{\Delta_{k}^{n} \tau \geq t_{0}\right\}}\right] .
\end{aligned}
$$


Putting the previous estimates together and using (2.9) and Lemma 2.1(i),

$$
\limsup _{n \rightarrow \infty}\left|\mathrm{E}\left\{\tilde{\beta}_{n}^{t}(\varphi)-\tilde{\beta}_{n}(\varphi)\right\}\right| \leq 2\left(\int|\varphi(x)| \mathbf{1}_{\left\{|\varphi(x)|>T_{0}\right\}} \nu(\mathrm{d} x) \vee T_{0}^{-1}\right),
$$

which can be made arbitrarily small by taking $T_{0}$ large enough.

Theorem 2.1. Under the conditions of Proposition 2.1 and Lemma 2.1(i), the statistics $\tilde{\beta}_{n}(\varphi)$ in (2.2) are consistent estimators for $\breve{\beta}(\varphi)$ in (2.7).

Proof. We apply arguments similar to the weak law of large numbers for row-wise independent arrays described in, e.g. [7, Theorem 5.2.3]. (The results in Chung [7, pp. 112-121] are proved for independent and identically distributed random variables, but they can be readily extended to row-wise independent arrays.) Let $\varepsilon>0$. We first note that in light of Lemma 2.1 and Proposition 2.1, there exists $n_{0}>0$ such that

$$
\left|\mathrm{E} \tilde{\beta}_{n}^{t}(\varphi)-\breve{\beta}(\varphi)\right|<\frac{\varepsilon}{2}
$$

for any $n \geq n_{0}$. Next, assuming that (2.16) holds and noticing that $\tilde{\beta}_{n}(\varphi)=\tilde{\beta}_{n}^{t}(\varphi)$ on the event $E=\left\{\left|\varphi\left(Z_{\tau_{k}^{n}}-Z_{\tau_{k-1}^{n}}\right)\right| \leq \tau_{n}^{n}\right.$ for all $\left.k\right\}$,

$$
\begin{aligned}
\mathrm{P}\left(\left|\tilde{\beta}_{n}(\varphi)-\breve{\beta}(\varphi)\right|>\varepsilon\right) & \leq \mathrm{P}\left(\left|\tilde{\beta}_{n}(\varphi)-\breve{\beta}(\varphi)\right|>\varepsilon, E^{\mathrm{c}}\right)+\mathrm{P}\left(\left|\tilde{\beta}_{n}^{t}(\varphi)-\breve{\beta}(\varphi)\right|>\varepsilon, E\right) \\
& \leq \mathrm{P}\left(E^{\mathrm{c}}\right)+\mathrm{P}\left(\left|\tilde{\beta}_{n}^{t}(\varphi)-\breve{\beta}(\varphi)\right|>\varepsilon\right) \\
& \leq \mathrm{P}\left(E^{\mathrm{c}}\right)+\mathrm{P}\left(\left|\tilde{\beta}_{n}^{t}(\varphi)-\mathrm{E} \tilde{\beta}_{n}^{t}(\varphi)\right|>\frac{\varepsilon}{2}\right)
\end{aligned}
$$

Using Chebyshev's inequality, we obtain, for $n \geq n_{0}$,

$$
\mathrm{P}\left(\left|\tilde{\beta}_{n}(\varphi)-\breve{\beta}(\varphi)\right|>\varepsilon\right) \leq B_{n}+\frac{4}{\varepsilon^{2}} C_{n},
$$

where

$$
B_{n}:=\sum_{k=1}^{n} \mathrm{P}\left(\left|\varphi\left(Z_{\tau_{k}^{n}}-Z_{\tau_{k-1}^{n}}\right)\right|>\tau_{n}^{n}\right), \quad C_{n}:=\operatorname{var}\left(\tilde{\beta}_{n}^{t}(\varphi)\right) .
$$

We show that $B_{n}$ and $C_{n}$ vanish. First, using Markov's inequality, under the convention that $0 / 0=0$,

$$
B_{n} \leq \mathrm{E}\left[\frac{1}{\tau_{n}^{n}} \sum_{k=1}^{n}|\varphi|\left(Z_{\tau_{k}^{n}}-Z_{\tau_{k-1}^{n}}\right) \mathbf{1}_{\left\{|\varphi|\left(Z_{\tau_{k}^{n}}-Z_{\tau_{k-1}^{n}}\right)>\tau_{n}^{n}\right\}}\right]
$$

which is the expression on the right-hand side of (2.14) and, hence, it can be proved to converge to 0 along the same lines as in the proof of Lemma 2.1 .

Next, using the law of total variance, conditioning on $\tau_{.}^{n}=\left(\tau_{1}^{n}, \ldots, \tau_{n}^{n}\right)$,

$$
\operatorname{var}\left(\tilde{\beta}_{n}^{t}(\varphi)\right)=\operatorname{var}\left(\mathrm{E}\left[\tilde{\beta}_{n}^{t}(\varphi) \mid \tau_{.}^{n}\right]\right)+\mathrm{E}\left[\operatorname{var}\left(\tilde{\beta}_{n}^{t}(\varphi) \mid \tau_{.}^{n}\right)\right]
$$


Let us denote by $D_{n}$ and $E_{n}$ the two terms on the right-hand side of (2.18). Conditioning on $\tau_{.}^{n}$, the terms in (2.11) are independent, and we can write

$$
\begin{aligned}
& D_{n}=\operatorname{var}\left(\left.\frac{1}{\tau_{n}^{n}} \sum_{k=1}^{n} \mathrm{E}\left[\varphi\left(Z_{t}\right) \mathbf{1}_{\left\{\left|\varphi\left(Z_{t}\right)\right| \leq s\right\}}\right]\right|_{t=\Delta_{k}^{n} \tau, s=\tau_{n}^{n}}\right), \\
& E_{n} \leq \mathrm{E}\left[\left.\frac{1}{\left(\tau_{n}^{n}\right)^{2}} \sum_{k=1}^{n} \mathrm{E}\left[\varphi^{2}\left(Z_{t}\right) \mathbf{1}_{\left\{\left|\varphi\left(Z_{t}\right)\right| \leq s\right\}}\right]\right|_{t=\Delta_{k}^{n} \tau, s=\tau_{n}^{n}}\right] .
\end{aligned}
$$

Clearly,

$$
\begin{aligned}
D_{n} \leq & 2 \operatorname{var}\left(\left.\frac{1}{\tau_{n}^{n}} \sum_{k=1}^{n} \mathrm{E}\left[\varphi\left(Z_{t}\right) \mathbf{1}_{\left\{\left|\varphi\left(Z_{t}\right)\right|>s\right\}}\right]\right|_{t=\Delta_{k}^{n} \tau, s=\tau_{n}^{n}}\right) \\
& +2 \operatorname{var}\left(\frac{1}{\tau_{n}^{n}} \sum_{k=1}^{n} H_{\varphi}\left(\Delta_{k}^{n} \tau\right)-\breve{\beta}(\varphi)\right),
\end{aligned}
$$

where, as before, $H_{\varphi}(t):=\mathrm{E}\left[\varphi\left(Z_{t}\right)\right]$. Using the inequality $\operatorname{var}(X) \leq E X^{2}$, estimate (2.8), and the fact that $\tau_{n}^{n}=\sum_{k=1}^{n} \tau_{k}^{n}$, the term on the right-hand side of (2.21) can be bounded by

$$
M_{\varphi} \mathrm{E}\left[\left.\frac{1}{\tau_{n}^{n}} \sum_{k=1}^{n} \mathrm{E}\left[|\varphi|\left(Z_{t}\right) \mathbf{1}_{\left\{\left|\varphi\left(Z_{t}\right)\right|>s\right\}}\right]\right|_{t=\Delta_{k}^{n} \tau, s=\tau_{n}^{n}}\right],
$$

which again converges to 0 by the same arguments following (2.14) in Lemma 2.1. Again, using $\operatorname{var}(X) \leq E X^{2},(2.8)$, and $\tau_{n}^{n}=\sum_{k=1}^{n} \tau_{k}^{n}$, the term in line (2.22) can be bounded as follows for any fixed $\varepsilon>0$ :

$$
\begin{aligned}
\left(M_{\varphi}\right. & +|\breve{\beta}(\varphi)|) \mathrm{E} \frac{1}{\tau_{n}^{n}} \sum_{k=1}^{n}\left|\frac{1}{\Delta_{k}^{n} \tau} H_{\varphi}\left(\Delta_{k}^{n} \tau\right)-\breve{\beta}(\varphi)\right| \Delta_{k}^{n} \tau \\
& \leq\left(M_{\varphi}+|\breve{\beta}(\varphi)|\right)^{2} \mathrm{E} \frac{1}{\tau_{n}^{n}} \sum_{k=1}^{n} \Delta_{k}^{n} \tau \mathbf{1}_{\left\{\Delta_{k}^{n} \tau \geq t_{0}\right\}}+\left(M_{\varphi}+|\breve{\beta}(\varphi)|\right) \varepsilon,
\end{aligned}
$$

where $t_{0}:=t_{0}(\varepsilon)$ is chosen such that $\left|H_{\varphi}(t) / t-\breve{\beta}(\varphi)\right| \leq \varepsilon$. In view of (2.9) and since $\varepsilon>0$ is arbitrary, we conclude that term (2.22) converges to 0 and so does $D_{n}$.

We now prove that the term on the right-hand side of (2.20) converges to 0 . We shall use the inequality

$$
\mathrm{E}|Z|^{2} \mathbf{1}_{\{|Z| \leq s\}} \leq 2 s \int_{(0,1)} \mathrm{E}\left[|Z| \mathbf{1}_{\{|Z|>u s\}}\right] \mathrm{d} u,
$$

which can be easily deduced as follows:

$$
\begin{aligned}
\mathrm{E}|Z|^{2} \mathbf{1}_{\{|Z| \leq s\}} & =2 \int_{0}^{s} v \mathrm{P}(v<|Z| \leq s) \mathrm{d} v \\
& \leq 2 \int_{0}^{s} v \mathrm{P}(|Z|>v) \mathrm{d} v \\
& \leq 2 s^{2} \int_{0}^{1} u \mathrm{P}(|Z|>u s) \mathrm{d} u \\
& \leq 2 s \int_{0}^{1} \mathrm{E}\left[|Z| \mathbf{1}_{\{|Z|>u s\}}\right] \mathrm{d} u .
\end{aligned}
$$


Applying (2.23) to each term in (2.20), $E_{n}$ can be bounded by

$$
\mathrm{E}\left[\left.\frac{2}{\tau_{n}^{n}} \sum_{k=1}^{n} \int_{0}^{1} \mathrm{E}\left[\left|\varphi\left(Z_{t}\right)\right| \mathbf{1}_{\left\{\left|\varphi\left(Z_{t}\right)\right|>u s\right\}}\right]\right|_{t=\Delta_{k}^{n} \tau, s=\tau_{n}^{n}} \mathrm{~d} u\right] \leq 2 \int_{0}^{1} s_{n}(u) \mathrm{d} u,
$$

where

$$
s_{n}(u):=\mathrm{E}\left[\left.\frac{1}{\tau_{n}^{n}} \sum_{k=1}^{n} \mathrm{E}\left[\left|\varphi\left(Z_{t}\right)\right| \mathbf{1}_{\left\{\left|\varphi\left(Z_{t}\right)\right|>u s\right\}}\right]\right|_{t=\Delta_{k}^{n} \tau, s=\tau_{n}^{n}}\right] .
$$

Note that $s_{n}(u) \leq M_{\varphi}$ for all $u \in[0,1]$. Fix $u_{0} \in(0,1)$ and $0<T_{0}<\infty$. There exists $t_{0}:=t_{0}\left(u_{0}, T_{0}\right)$ such that

$$
0 \leq \mathrm{E}\left[\left|\varphi\left(Z_{t}\right)\right| \mathbf{1}_{\left\{\left|\varphi\left(Z_{t}\right)\right|>u_{0} T_{0}\right\}}\right] \leq 2 t \int|\varphi(x)| \mathbf{1}_{\left\{|\varphi(x)|>u_{0} T_{0}\right\}} v(\mathrm{~d} x)
$$

for all $0<t<t_{0}$. Using the fact that

$$
\mathbf{1}_{\left\{\left|\varphi\left(Z_{t}\right)\right|>u s\right\}} \leq \mathbf{1}_{\left\{s \leq T_{0}\right\}}+\mathbf{1}_{\left\{\left|\varphi\left(Z_{t}\right)\right|>u_{0} T_{0}\right\}}
$$

for any $u \geq u_{0}$, we have

$$
s_{n}(u) \leq 2 M_{\varphi} \mathrm{P}\left(\tau_{n}^{n} \leq T_{0}\right)+\mathrm{E}\left[\left.\frac{2}{\tau_{n}^{n}} \sum_{k=1}^{n} \mathrm{E}\left[\left|\varphi\left(Z_{t}\right)\right| \mathbf{1}_{\left\{\left|\varphi\left(Z_{t}\right)\right|>u_{0} T_{0}\right\}}\right]\right|_{t=\Delta_{k}^{n} \tau}\right]
$$

for $u \geq u_{0}$. Next, by breaking up the above summation into those $k$ for which $\Delta_{k}^{n} \geq t_{0}$ and those for which $\Delta_{k}^{n}<t_{0}$, and using the fact that $\mathrm{E}\left[\left|\varphi\left(Z_{t}\right)\right|\right] \leq M_{\varphi} t$ and (2.25), for any $u_{0} \leq u \leq 1$,

$$
s_{n}(u) \leq 2 M_{\varphi} \mathrm{P}\left(\tau_{n}^{n} \leq T_{0}\right)+2 M_{\varphi} \mathrm{E} \frac{1}{\tau_{n}^{n}} \sum_{k=1}^{n} \Delta_{k}^{n} \tau \mathbf{1}_{\left\{\Delta_{k}^{n} \tau \geq t_{0}\right\}}+4 \int|\varphi| \mathbf{1}_{\left\{|\varphi|>u_{0} T_{0}\right\}} \mathrm{d} \nu .
$$

Breaking the integral in (2.24),

$$
\begin{aligned}
E_{n} \leq & 2 u_{0} M_{\varphi}+2 M_{\varphi} \mathrm{E} \frac{1}{\tau_{n}^{n}} \sum_{k=1}^{n} \Delta_{k}^{n} \tau \mathbf{1}_{\left\{\Delta_{k}^{n} \tau \geq t_{0}\right\}}+2 M_{\varphi} \mathrm{P}\left(\tau_{n}^{n} \leq T_{0}\right) \\
& +4 \int|\varphi(x)| \mathbf{1}_{\left\{|\varphi(x)|>u_{0} T_{0}\right\}} v(\mathrm{~d} x) .
\end{aligned}
$$

In view of Proposition 2.1(ii) and Lemma 2.1(i),

$$
\limsup _{n \rightarrow \infty} E_{n} \leq 2 u_{0} M_{\varphi}+4 \int|\varphi(x)| \mathbf{1}_{\left\{|\varphi(x)|>u_{0} T_{0}\right\}} v(\mathrm{~d} x),
$$

which can be made arbitrarily small by taking small enough $u_{0}$ and large enough $T_{0}$. This proves that the second term on the right-hand side of (2.18), and thereof (2.17), vanishes as $n \rightarrow \infty$.

Remark 2.1. 1. Clearly, (2.8) will be satisfied if Conditions 2.1 hold and $\varphi$ is bounded. Moreover, (2.8) holds as well, if $\varphi$ has linear growth, Conditions 2.1 are satisfied, and $Z$ has finite 
first moment. Indeed, in light of (2.6), there exists $t_{0}>0$ such that $\sup _{0<t<t_{0}} \mathrm{E}\left|\varphi\left(Z_{t}\right)\right| / t<\infty$. Suppose that $|\varphi(x)| \leq c|x|$, whenever $|x|>x_{0}$ for some $x_{0}>0$. Then,

$$
\sup _{t \geq t_{0}} \frac{1}{t} \mathrm{E}\left|\varphi\left(Z_{t}\right)\right| \leq c \sup _{t \geq t_{0}} \frac{1}{t} \mathrm{E}\left|Z_{t}\right|+\frac{1}{t_{0}} \sup _{|x| \leq x_{0}}|\varphi(x)| .
$$

Hence, it remains to show that the first term on the right-hand side of the above inequality is bounded. This follows from the inequality

$$
\mathrm{E}\left|Z_{t}\right| \leq\lfloor t\rfloor \mathrm{E}\left|Z_{1}\right|+\mathrm{E} Z_{1}^{*},
$$

where $Z_{1}^{*}:=\sup _{t \leq 1}\left|Z_{t}\right|$. Here $\mathrm{E} Z_{1}^{*}<\infty$ in light of Theorem 25.18 of [21], which is actually stated for submultiplicative moment functions $g$, but which can be readily modified to cover subadditive $g$ as well.

2. Suppose now that $\varphi$ has quadratic growth, Conditions 2.1 are satisfied, and that $Z$ has finite second moment. In this case, there exist constants $c_{1}, c_{2}>0$ such that

$$
\mathrm{E}\left|\varphi\left(Z_{t}\right)\right| \leq c_{1} t+c_{2} t^{2}
$$

for all $t>0$. Proposition 2.1 and Lemma 2.1 hold true if we impose Lemma 2.1(i) and the following condition instead of (2.9):

$$
\lim _{n \rightarrow \infty} \mathrm{E}\left[\frac{1}{\tau\left(t_{n}^{n}\right)} \sum_{k=1}^{n}\left(\Delta_{k}^{n} \tau\right)^{2} \mathbf{1}_{\left\{\Delta_{k}^{n} \tau \geq t_{0}\right\}}\right]=0
$$

for all $t_{0}>0$. For Theorem 2.1 to hold true, Proposition 2.1(ii) suffices and, for any $t_{0}>0$,

$$
\lim _{n \rightarrow \infty} \mathrm{E}\left[\frac{1}{\tau\left(t_{n}^{n}\right)} \sum_{k=1}^{n}\left(\Delta_{k}^{n} \tau\right)^{3} \mathbf{1}_{\left\{\Delta_{k}^{n} \tau \geq t_{0}\right\}}\right]=0 .
$$

More precisely, when trying to show that the different terms of (2.17) vanish, the following limit naturally appears:

$$
\lim _{n \rightarrow \infty} \mathrm{E}\left[\frac{1}{\tau\left(t_{n}^{n}\right)} \sum_{k=1}^{n}\left(\Delta_{k}^{n} \tau\right)^{2} \mathbf{1}_{\left\{\Delta_{k}^{n} \tau \geq t_{0}\right\}}\right]^{2},
$$

which can be linked to (2.26) in view of Jensen's inequality:

$$
\left(\frac{1}{\tau_{n}^{n}} \sum_{k=1}^{n} c_{k} \Delta_{k}^{n}\right)^{2} \leq \frac{1}{\tau_{n}^{n}} \sum_{k=1}^{n} c_{k}^{2} \Delta_{k}^{n} \quad \text { a.s. }
$$

We complete this section with some remarks concerning the pure Lévy model where $\tau(t) \equiv t$ and $X=Z$. This case was studied in [24] (see their Theorem 5.1) for regular sampling schemes and functions $\varphi$ satisfying certain regularity conditions. In light of the results of this section, under the pure Lévy model, $\hat{\beta}_{n}(\varphi)=\tilde{\beta}_{n}(\varphi)$ and Proposition 2.1(ii) is satisfied whenever $\bar{\delta}^{n}:=\max _{k}\left\{t_{k+1}^{n}-t_{k}^{n}\right\} \rightarrow 0$. Thus, $\hat{\beta}_{n}(\varphi) \stackrel{\mathrm{P}}{\rightarrow} \breve{\beta}(\varphi)$, provided that $T_{n} \rightarrow \infty, \bar{\delta}^{n} \rightarrow 0$, and $\varphi$ satisfies Proposition 2.1(i). It turns out that (2.8) is not needed, as the following result shows. Its proof is similar to that of Theorem 2.1 and is thus omitted (see [14] for the details).

Proposition 2.2. Let $X$ be a Lévy process with triplet $\left(b, \sigma^{2}, v\right)$, let $\varphi$ be a function satisfying Conditions 2.1, and let $\hat{\beta}^{\pi}(\varphi)$ be estimator (1.5). Then, for any $0<\varepsilon<1$, there exist $T<\infty$ and $\delta>0$ such that $\mathrm{P}\left(\left|\hat{\beta}^{\pi}(\varphi)-\breve{\beta}(\varphi)\right|>\varepsilon\right)<\varepsilon$, whenever $t_{n}>T$ and $\max _{k}\left(t_{k}-t_{k-1}\right)<\delta$. 


\section{Random clocks driven by ergodic diffusions}

In this section we consider random clocks $\{\tau(t)\}_{t \geq 0}$ of the form (1.4) with $r(t):=g(\tilde{r}(t))$, where $g$ is a nonnegative function and $\left\{\tilde{r}_{t}\right\}_{t \geq 0}$ is an ergodic diffusion process; that is, $\{\tilde{r}(t)\}_{t \geq 0}$ is a regular recurrent strong Markov process with continuous paths taking values on an interval $I=(a, b) \subset \mathbb{R}$ (see, e.g. [17]). As explained in the introduction, mean-reverting processes $\tilde{r}$ and monotone continuous functions $g$ are especially attractive since in this case the resulting time-changed Lévy process $X(t):=Z_{\tau(t)}$ will exhibit the volatility clustering effect.

Proposition 3.1. Consider the model (1.3)-(1.4) under the following setting:

(a) $Z$ is a Lévy process with Lévy triplet $\left(\sigma^{2}, b, v\right)$;

(b) the instantaneous rate process $r$ is independent of $Z$ and is given by $r(t):=g(\tilde{r}(t))$ for a measurable nonnegative function $g$ and an ergodic diffusion $\left\{\tilde{r}_{t}\right\}_{t \geq 0}$ with

$$
m_{2}(g):=\sup _{t \geq 0} \mathrm{E}^{2}(\tilde{r}(t))<\infty
$$

and invariant measure $\zeta$ satisfying $\bar{\zeta}(g):=\int g(x) \zeta(\mathrm{d} x) \in(0, \infty)$.

Then, the statistics

$$
\hat{\beta}_{n}(\varphi):=\frac{1}{T_{n}} \sum_{k=1}^{n} \varphi\left(X_{t_{k}^{n}}-X_{t_{k-1}^{n}}\right)
$$

are consistent and asymptotically unbiased estimators for $\bar{\zeta}(g) \breve{\beta}(\varphi)$ when $T_{n} \rightarrow \infty$ and $\bar{\delta}^{n}:=$ $\max _{k}\left(t_{k}^{n}-t_{k-1}^{n}\right) \rightarrow 0$, provided that

(i) $\varphi$ is a continuous function satisfying Conditions 2.1 and (2.8);

(ii) (2.9) holds.

Proof. Note that

$$
\hat{\beta}_{n}(\varphi)=\tilde{\beta}_{n}(\varphi) \frac{\int_{0}^{T_{n}} g(\tilde{r}(u)) \mathrm{d} u}{T_{n}} .
$$

By the ergodic theorem (1.11), the last factor converges a.s. to $\bar{\zeta}(g)$ and, hence, Lemma 2.1(i) is satisfied. Consistency is now clear in light of Theorem 2.1. For unbiasedness, first note that $\bar{r}(t):=\int_{0}^{t} r(u) \mathrm{d} u / t$ is uniformly integrable since

$$
\sup _{t>0} \mathrm{E}\left(\frac{1}{t} \int_{0}^{t} r(u) \mathrm{d} u\right)^{2} \leq \sup _{t>0} \frac{1}{t} \int_{0}^{t} \mathrm{E} g^{2}(r(u)) \mathrm{d} u \leq m_{2}(g)<\infty .
$$

Also, by the ergodic theorem $(1.11), \lim _{t \rightarrow \infty} \bar{r}(t)=\bar{\zeta}(g)$ a.s., and, thus,

$$
\lim _{t \rightarrow \infty} \mathrm{E}|\bar{r}(t)-\bar{\zeta}(g)|=0 .
$$

Next, we write

$$
\mathrm{E} \hat{\beta}_{n}(\varphi)=\bar{\zeta}(g) \mathrm{E} \tilde{\beta}_{n}(\varphi)+\mathrm{E}\left[\left(\frac{\tau_{n}^{n}}{t_{n}^{n}}-\bar{\zeta}(g)\right) \frac{1}{\tau_{n}^{n}} \sum_{k=1}^{n} H_{\varphi}\left(\Delta_{k}^{n} \tau\right)\right]
$$


The first term on the right-hand side converges to $\bar{\zeta}(g) \breve{\beta}(\varphi)$, while the absolute value of the second term is bounded by

$$
M_{\varphi} \mathrm{E}\left|\frac{\tau_{n}^{n}}{t_{n}^{n}}-\bar{\zeta}(g)\right|=M_{\varphi} \mathrm{E}\left|\bar{r}\left(t_{n}^{n}\right)-\bar{\zeta}(g)\right| \rightarrow 0 \quad \text { as } n \rightarrow \infty .
$$

In the case that $g$ is bounded, condition (2.9), condition (3.1), and $\bar{\zeta}(g)<\infty$ hold automatically, and, thus, the following two limits are true:

$$
\hat{\beta}_{n}(\varphi) \stackrel{\mathrm{P}}{\rightarrow} \bar{\zeta} \breve{\beta}(\varphi), \quad \mathrm{E} \hat{\beta}_{n}(\varphi) \rightarrow \bar{\zeta} \beta(\varphi), \quad \text { as } n \rightarrow \infty .
$$

Given that $r(t)=g(\tilde{r})$ plays the role of volatility, we could argue that there is no reason to assume that the volatility will take arbitrarily large values, and, thus, the boundedness assumption for $g$ is not completely implausible. Nevertheless, since an upper bound for $g$ cannot be determined in principle, it is natural to consider the case $g(x)=x \mathbf{1}_{\{x \geq 0\}}$. Note that in this case, for (3.1) to hold, it suffices that

$$
m_{2}:=\sup _{t \geq 0} \mathrm{E} \tilde{r}^{2}(t)<\infty .
$$

The following lemma gives a useful sufficient condition for (2.9) to hold.

Lemma 3.1. Under the setting of Proposition 3.1(a)-(b) with $g(x):=x \mathbf{1}_{\{x \geq 0\}}$, condition (2.9) is satisfied if

$$
\lim _{m \rightarrow \infty} \limsup _{n \rightarrow \infty} \mathrm{P}\left(\text { there exists } k \text { such that } \sup _{t \in I_{k}^{n}}\left|\tilde{r}(t)-\tilde{r}\left(t_{k-1}^{n}\right)\right| \geq \frac{1}{2}\left(\frac{t_{0}}{\delta_{k}^{n}}-m\right)\right)=0
$$

for any $t_{0}, m>0$, where $I_{k}^{n}:=\left[t_{k-1}^{n}, t_{k}^{n}\right]$ and $\delta_{k}^{n}:=t_{k-1}^{n}-t_{k}^{n}$.

Proof. Fix $m>0$. For $n \geq 1$, let $B_{n}^{m}:=\left\{k \in\{1, \ldots, n\}: \sup _{t \in\left[t_{k-1}^{n}, t_{k}^{n}\right]} r(t)<m\right\}$. Clearly,

$$
\limsup _{n \rightarrow \infty} \mathrm{E} \frac{1}{\tau_{n}^{n}} \sum_{k \in B_{n}^{m}}\left(\Delta_{k}^{n} \tau\right) \mathbf{1}_{\left\{\Delta_{k}^{n} \tau \geq t_{0}\right\}} \leq \limsup _{n \rightarrow \infty} \mathrm{E} \frac{1}{\tau_{n}^{n}} \sum_{k \in B_{n}^{m}}\left(\Delta_{k}^{n} \tau\right) \mathbf{1}_{\left\{\bar{\delta}^{n} \geq t_{0} / m\right\}}=0,
$$

where the last limit follows from the fact that $\bar{\delta}^{n} \rightarrow 0$. Next, let

$$
C_{n}^{m}:=\left\{k \in\{1, \ldots, n\}: \inf _{t \in\left[t_{k-1}^{n}, t_{k}^{n}\right]} \tilde{r}(t) \geq m\right\} .
$$

Then,

$$
\begin{aligned}
\limsup _{n \rightarrow \infty} \mathrm{E} \frac{1}{\tau_{n}^{n}} \sum_{k \in C_{n}^{m}}\left(\Delta_{k}^{n} \tau\right) \mathbf{1}_{\left\{\Delta_{k}^{n} \tau \geq t_{0}\right\}} & \leq \limsup _{n \rightarrow \infty} \mathrm{E} \frac{\int_{0}^{T_{n}} \tilde{r}(u) \mathbf{1}_{\{\tilde{r}(u) \geq m\}} \mathrm{d} u}{\int_{0}^{T_{n}} \tilde{r}(u) \mathbf{1}_{\{\tilde{r}(u) \geq 0\}} \mathrm{d} u}, \\
& =\frac{\int_{m}^{\infty} x \zeta(\mathrm{d} x)}{\int_{0}^{\infty} x \zeta(\mathrm{d} x)},
\end{aligned}
$$

by the dominated convergence theorem and the ergodic theorem (1.11). Next, let $D_{n}^{m}:=\{k \in$ $\{1, \ldots, n\}$ : there exist $u, v \in\left[t_{k-1}^{n}, t_{k}^{n}\right]$ with $\left.\tilde{r}(u)<m<\tilde{r}(v)\right\}$. Now, if $k \in D_{n}^{m}$ is such that $\Delta_{k}^{n} \tau>t_{0}$ then $\sup _{t \in\left[t_{k-1}^{n}, t_{k}^{n}\right]} \tilde{r}(t) \geq t_{0} / \delta_{k}^{n}$, and, thus, the following inequalities must be true:

$$
\sup _{t \in\left[t_{k-1}^{n}, t_{k}^{n}\right]}\left|\tilde{r}(t)-\tilde{r}\left(t_{k-1}^{n}\right)\right| \geq \frac{1}{2}\left(\frac{t_{0}}{\delta_{k}^{n}}-m\right) .
$$


If $\Omega_{n}^{m}$ denotes the event that there exists a $k$ satisfying the above inequality then

$$
\limsup _{n \rightarrow \infty} \mathrm{E} \frac{1}{\tau_{n}^{n}} \sum_{k \in D_{n}^{m}}\left(\Delta_{k}^{n} \tau\right) \mathbf{1}_{\left\{\Delta_{k}^{n} \tau \geq t_{0}\right\}} \leq \limsup _{n \rightarrow \infty} \mathrm{P}\left(\Omega_{n}^{m}\right) .
$$

Putting together the previous estimates, for each $m>0$,

$$
\limsup _{n \rightarrow \infty} \mathrm{E} \frac{1}{\tau_{n}^{n}} \sum_{k=1}^{n}\left(\Delta_{k}^{n} \tau\right) \mathbf{1}_{\left\{\Delta_{k}^{n} \tau \geq t_{0}\right\}} \leq \frac{\int_{m}^{\infty} x \zeta(\mathrm{d} x)}{\int_{0}^{\infty} x \zeta(\mathrm{d} x)}+\limsup _{n \rightarrow \infty} \mathrm{P}\left(\Omega_{n}^{m}\right) .
$$

We finally make $m \rightarrow \infty$.

The most well-known examples of diffusions are solutions to stochastic differential equations of the form

$$
\mathrm{d} \tilde{r}(t)=b(\tilde{r}(t)) \mathrm{d} t+\sigma(\tilde{r}(t)) \mathrm{d} W_{t} .
$$

Two important instances of mean-reverting diffusions of this kind are the Ornstein-Uhlenbeck process and CIR processes (see Examples 3.1 and 3.2, below). Conditions for the solution of (3.5) to be ergodic can be found in, e.g. [23]. We will make use of moment estimates for (3.5) in order to conclude the sufficient conditions of Lemma 3.1. Under a linear growth condition of the form

$$
|b(x)|+|\sigma(x)| \leq K(1+|x|)
$$

for all $x$ and a certain constant $K<\infty$, it turns out that

$$
\mathrm{E}\left[\sup _{0 \leq s \leq h}\left|\tilde{r}\left(s_{0}+s\right)-\tilde{r}\left(s_{0}\right)\right|^{2 m}\right] \leq k_{m} h^{m}\left(1+\mathrm{E}\left|\tilde{r}\left(s_{0}\right)\right|^{2 m}\right) \mathrm{e}^{k_{m} h}
$$

for any $s_{0} \geq 0,0<h \leq 1$, and $m \geq 1$, where $k_{m}$ is a constant depending only on $m$ and $K$. We present the proof of the above estimate in Appendix A for the sake of completeness. We are now ready to establish the consistency of the estimators in (3.2).

Proposition 3.2. Under the setting of Proposition 3.1(a)-(b) with $g(x):=x \mathbf{1}_{\{x \geq 0\}}$, suppose also that

(b') $\tilde{r}$ satisfies (3.5) with the linear growth condition (3.6).

Then, the statistics $\hat{\beta}_{n}(\varphi)$ in (3.2) are both consistent and asymptotically unbiased estimators for the parameter $\bar{\zeta} \tilde{\beta}(\varphi)$ with $\bar{\zeta}:=\int_{0}^{\infty} x \zeta(\mathrm{d} x)$, provided that Proposition 3.1(i) holds and also that $T_{n} \rightarrow \infty$ and $T_{n}\left(\bar{\delta}^{n}\right)^{2} \rightarrow 0$ as $n \rightarrow \infty$.

Proof. From Propositions 3.1 and Lemma 3.1, it suffices to prove that, for all $t_{0}, m>0$,

$$
\lim _{n \rightarrow \infty} \sum_{k=1}^{n} \mathrm{P}\left(\sup _{t \in I_{k}^{n}}\left|\tilde{r}(t)-\tilde{r}\left(t_{k-1}^{n}\right)\right| \geq \frac{1}{2}\left(\frac{t_{0}}{\Delta_{k}^{n}}-m\right)\right)=0 .
$$

Let $n$ be large enough such that $\bar{\delta}^{n}<t_{0} /(2 m)$, and write $c_{k}^{n}=\left(t_{0}-m \delta_{k}^{n}\right) / 2$ and $\kappa=4 / t_{0}^{2}$. 
Using the bound in (3.7), we can find a constant $K$ such that

$$
\begin{aligned}
\sum_{k=1}^{n} \mathrm{P}\left(\sup _{t \in I_{k}^{n}}\left|\tilde{r}(t)-\tilde{r}\left(t_{k-1}^{n}\right)\right| \geq \frac{c_{k}^{n}}{\delta_{k}^{n}}\right) & \leq \kappa \sum_{k=1}^{n}\left(\delta_{k}^{n}\right)^{2} \mathrm{E}\left[\sup _{t \in I_{k}^{n}}\left|\tilde{r}(t)-\tilde{r}\left(t_{k-1}^{n}\right)\right|^{2}\right] \\
& \leq(\kappa)\left(k_{2}\right) \sum_{k=1}^{n}\left(\delta_{k}^{n}\right)^{3}\left(1+\mathrm{E} \tilde{r}^{2}\left(t_{k-1}^{n}\right)\right) \mathrm{e}^{k_{2} \delta_{k}^{n}} \\
& \leq K \sum_{k=1}^{n}\left(\delta_{k}^{n}\right)^{3} \leq K\left(\bar{\delta}^{n}\right)^{2} T_{n} \rightarrow 0 \quad \text { as } n \rightarrow \infty
\end{aligned}
$$

Example 3.1. For positive $\alpha, v$, and $m$, consider the mean-reverting CIR process

$$
\mathrm{d} r(t)=\alpha(m-r(t)) \mathrm{d} t+v \sqrt{r(t)} \mathrm{d} W_{t},
$$

where $\left\{W_{t}\right\}_{t \geq 0}$ is a standard Brownian motion independent of the Lévy process $X$ and $\alpha m / v^{2}>\frac{1}{2}$. The equation above has a weak nonnegative solution with unique positive stationary distribution $\Gamma\left(2 m \alpha / v^{2}, v^{2} / 2 \alpha\right)$. Also, the conditional mean and variance given $r(0)$ are determined by

$$
\begin{gathered}
\mathrm{E}[r(t) \mid r(0)]=r(0) \mathrm{e}^{-\alpha t}+m\left(1-\mathrm{e}^{-\alpha t}\right), \\
\operatorname{var}(r(t) \mid r(0))=r(0) \frac{v^{2}}{\alpha}\left(\mathrm{e}^{-\alpha t}-\mathrm{e}^{-2 \alpha t}\right)+m \frac{v^{2}}{2 \alpha}\left(1-\mathrm{e}^{-\alpha t}\right)^{2} .
\end{gathered}
$$

Clearly, this equation satisfies the linear growth condition (3.6) and all the conditions of Proposition 3.2. Then, $\tilde{\beta}_{n}(\varphi)$ is an asymptotically unbiased estimator of $\breve{\beta}(\varphi)$, and the $\hat{\beta}_{n}(\varphi)$ in (3.2) are asymptotically consistent and unbiased estimators of $m \breve{\beta}$.

Example 3.2. Consider the mean-reverting Ornstein-Uhlenbeck process determined by the stochastic differential equation

$$
\mathrm{d} \tilde{r}(t)=\alpha(m-\tilde{r}(t)) \mathrm{d} t+v \sqrt{2 \alpha} \mathrm{d} W_{t},
$$

where $\left\{W_{t}\right\}_{t \geq 0}$ is a standard Brownian motion independent of the Lévy process $X$. The solution to $(3.8)$ is

$$
\tilde{r}(t)=m+(\tilde{r}(0)-m) \mathrm{e}^{-\alpha t}+v \sqrt{2 \alpha} \int_{0}^{t} \mathrm{e}^{-\alpha(t-s)} \mathrm{d} W_{s},
$$

and, thus, given $\tilde{r}(0), \tilde{r}(t)-\tilde{r}(0) \mathrm{e}^{-\alpha t} \sim \mathcal{N}\left(m\left(1-\mathrm{e}^{-\alpha t}\right), v^{2}\left(1-\mathrm{e}^{-2 \alpha t}\right)\right)$. Note that

$$
\lim _{t \rightarrow \infty} \mathrm{E} \tilde{r}^{2}(t)=v^{2}+m^{2}
$$

and the invariant distribution of $\tilde{r}$ is $\mathcal{N}\left(m, v^{2}\right)$. Let $b(x):=\alpha(m-x)$ be the drift, and let $\sigma:=v \sqrt{2 \alpha}$ be the diffusion of (3.8). Clearly, this equation satisfies the linear growth condition (3.6) and all the conditions of Proposition 3.2. Then, $\tilde{\beta}_{n}(\varphi)$ is an asymptotically unbiased estimator of $\breve{\beta}(\varphi)$, and the $\hat{\beta}_{n}(\varphi)$ in (3.2) are asymptotically consistent and unbiased estimators of $\mu \breve{\beta}$, where $\mu:=\mathrm{E}(v Z+m)_{+}$. 


\section{Consistency of the estimators for general sampling schemes}

A rather relevant question is whether the condition $T_{n}\left(\bar{\delta}^{n}\right)^{2} \rightarrow 0$ as $n \rightarrow \infty$ of Proposition 3.2 is actually necessary. This condition came from the path of proof we chose in working with the modified estimator (2.2). It is of interest to know whether or not we could directly apply the same reasonings to (3.2). We will discuss this point here. In short, we find that the following condition plays a similar role to (2.9) in this direction of proof:

$$
\lim _{n \rightarrow \infty} \mathrm{E}\left(\frac{1}{t_{n}^{n}} \sum_{k=1}^{n}\left(\Delta_{k}^{n} \tau\right) \mathbf{1}_{\left\{\Delta_{k}^{n} \tau \geq t_{0}\right\}}\right)^{2}=0
$$

We will show that, under the condition

$$
m_{2+\varepsilon}(g):=\sup _{t \geq 0} \mathrm{E}|g(\tilde{r}(t))|^{2+\varepsilon}<\infty
$$

for some $\varepsilon>0$, the rate $T_{n}\left(\bar{\delta}^{n}\right)^{2} \rightarrow 0$ as $n \rightarrow \infty$ is not needed.

Theorem 4.1. Consider the model (1.3)-(1.4) under the setting of Proposition 3.1(a)-(b) and also assuming that (4.2) holds for some $\varepsilon>0$. Then, the estimators in (3.2) are consistent and asymptotically unbiased for $\bar{\zeta}(g) \breve{\beta}(\varphi)$ when $T_{n} \rightarrow \infty$ and $\bar{\delta}^{n} \rightarrow 0$, provided that Proposition 3.1(i) is satisfied.

Proof. Let us assume for now that (4.1) is true. We shall see at the end of the proof that (4.2) implies (4.1) whenever $T_{n} \rightarrow \infty$ and $\bar{\delta}^{n} \rightarrow 0$. The proof is quite similar to that of Theorem 2.1 working with $\hat{\beta}_{n}$ instead of $\tilde{\beta}_{n}(\varphi)$ and with

$$
\hat{\beta}_{n}^{t}(\varphi):=\frac{1}{t_{n}^{n}} \sum_{k=1}^{n} \varphi\left(Z_{\tau\left(t_{k}^{n}\right)}-Z_{\tau\left(t_{k-1}^{n}\right)}\right) \mathbf{1}_{\left\{\left|\varphi\left(Z_{\tau\left(t_{k}^{n}\right)}-Z_{\tau\left(t_{k-1}^{n}\right)}\right)\right| \leq t_{n}^{n}\right\}}
$$

instead of $\tilde{\beta}_{n}^{t}$. We will outline the general steps. First, we check that $\hat{\beta}_{n}(\varphi)$ is asymptotically unbiased. This will follow because, conditioning on $\left\{\tau_{k}^{n}\right\}_{k \leq n}$,

$$
\begin{aligned}
\left|\mathrm{E} \hat{\beta}_{n}(\varphi)-\bar{\zeta} \breve{\beta}(\varphi)\right| \leq & \frac{1}{t_{n}^{n}} \mathrm{E} \sum_{k=1}^{n} \Delta_{k}^{n} \tau\left|\frac{1}{\Delta_{k}^{n} \tau} H_{\varphi}\left(\Delta_{k}^{n} \tau\right)-\breve{\beta}(\varphi)\right| \\
& +\breve{\beta}(\varphi)\left|\frac{1}{t_{n}^{n}} \mathrm{E} \int_{0}^{t_{n}^{n}} g(\tilde{r}(u)) \mathrm{d} u-\bar{\zeta}(g)\right|
\end{aligned}
$$

The second term on the right-hand side vanishes as $n \rightarrow \infty$ because of the ergodicity of $\tilde{r}$ and (3.1), similar to the verification of (3.3). The first term on the right-hand side can be bounded by

$$
\varepsilon \frac{1}{t_{n}^{n}} \mathrm{E} \int_{0}^{t_{n}^{n}} g(\tilde{r}(u)) \mathrm{d} u+\frac{c}{t_{n}^{n}} \mathrm{E} \sum_{k=1}^{n}\left(\Delta_{k}^{n} \tau\right) \mathbf{1}_{\left\{\Delta_{k}^{n} \tau \geq t_{0}\right\}}
$$

for any $\varepsilon>0$ and some $t_{0}=t_{0}(\varepsilon)$ such that $\left|H_{\varphi}(t) / t-\breve{\beta}(\varphi)\right|<\varepsilon$ for any $0<t<t_{0}$. The limit of the second term above converges to 0 in light of (4.1), while the first term converges to $\varepsilon \bar{\zeta}(g)$, which is arbitrarily small. The second step is to show that $\mathrm{E}\left[\hat{\beta}_{n}\{\varphi)-\hat{\beta}_{n}^{t}(\varphi)\right]=0$, which can be done almost identically to the proof of (2.12). The next step will be to bound 
$\mathrm{P}\left(\left|\hat{\beta}_{n}(\varphi)-\bar{\zeta} \breve{\beta}(\varphi)\right|>\varepsilon\right)$ as in (2.17) with $\tau_{n}^{n}$ and $\tilde{\beta}_{n}^{t}$ replaced by $t_{n}^{n}$ and $\hat{\beta}_{n}^{t}(\varphi)$, respectively, in the definition of $B_{n}$ and $C_{n}$. The limit of $B_{n}$ can be treated as before. For $C_{n}$, we use a decomposition similar to (2.18) with $D_{n}$ and $E_{n}$ being defined and bounded as in (2.19)-(2.20) with $\tau_{n}^{n}$ replaced by $t_{n}^{n}$. The convergence of $E_{n}$ to 0 can be proved in a similar manner to the proof of Theorem 2.1. The term

$$
D_{n}:=\operatorname{var}\left(\left.\frac{1}{t_{n}^{n}} \sum_{k=1}^{n} \mathrm{E}\left[\varphi\left(Z_{t}\right) \mathbf{1}_{\left\{\left|\varphi\left(Z_{t}\right)\right| \leq t_{n}^{n}\right\}}\right]\right|_{t=\Delta_{k}^{n} \tau}\right)
$$

requires some care. As before,

$$
\begin{aligned}
D_{n} \leq & 2 \mathrm{E}\left(\left.\frac{1}{t_{n}^{n}} \sum_{k=1}^{n} \mathrm{E}\left[|\varphi|\left(Z_{t}\right) \mathbf{1}_{\left.\left\{\left|\varphi\left(Z_{t}\right)\right|>t_{n}^{n}\right\}\right]}\right]\right|_{t=\Delta_{k}^{n} \tau}\right)^{2} \\
& +2 \operatorname{var}\left(\frac{1}{t_{n}^{n}} \sum_{k=1}^{n} H_{\varphi}\left(\Delta_{k}^{n} \tau\right)-\bar{\zeta}(g) \breve{\beta}(\varphi)\right) .
\end{aligned}
$$

Fix $T_{0}>0$, and let $t_{0}>0$ (depending on $T_{0}$ ) such that

$$
\mathrm{E}\left[|\varphi|\left(Z_{t}\right) \mathbf{1}_{\left\{\left|\varphi\left(Z_{t}\right)\right|>T_{0}\right\}}\right] \leq t\left(2 \int|\varphi| \mathbf{1}_{|\varphi| \geq T_{0}} \mathrm{~d} v \vee T_{0}^{-1}\right)
$$

for any $0<t<t_{0}$. Then, when $t_{n}^{n}>T_{0}$, the term in (4.3) can be bounded by

$$
c \mathrm{E}\left(\frac{1}{t_{n}^{n}} \int_{0}^{t_{n}^{n}} r(u) \mathrm{d} u\right)^{2}\left(\int|\varphi| \mathbf{1}_{|\varphi| \geq T_{0}} \mathrm{~d} \nu \vee T_{0}^{-1}\right)^{2}+c^{\prime} M_{\varphi} \mathrm{E}\left(\frac{1}{t_{n}^{n}} \sum_{k=1}^{n} \Delta_{k}^{n} \tau \mathbf{1}_{\left\{\Delta_{k}^{n} \tau \geq t_{0}\right\}}\right)^{2}
$$

for some constants $c, c^{\prime}>0$. The above bound converges to

$$
c \bar{\zeta}^{2}(g)\left(\int|\varphi| \mathbf{1}_{|\varphi| \geq T_{0}} \mathrm{~d} v \vee T_{0}^{-1}\right)^{2}
$$

in view of (4.1), the ergodicity of $\tilde{r}$, and (4.2). Making $T_{0} \rightarrow \infty$, we conclude that the term in (4.3) vanishes. The term in (4.4), which we denote by $F_{n}$, can be bounded in the following manner:

$$
\begin{aligned}
F_{n} \leq & 2 \mathrm{E}\left(\frac{1}{t_{n}^{n}} \sum_{k=1}^{n} \Delta_{k}^{n} \tau\left(\frac{1}{\Delta_{k}^{n} \tau} H_{\varphi}\left(\Delta_{k}^{n} \tau\right)-\breve{\beta}(\varphi)\right)\right)^{2} \\
& +2 \breve{\beta}(\varphi)^{2} \mathrm{E}\left(\frac{1}{t_{n}^{n}} \int_{0}^{t_{n}^{n}} r(u) \mathrm{d} u-\bar{\zeta}(g)\right)^{2} .
\end{aligned}
$$

The second term on the right-hand side above converges to 0 because of the ergodicity of $\tilde{r}$ and (4.2). For a fixed $\varepsilon$, the first term can be decomposed into two sums, when $\Delta_{k}^{n} \tau<t_{0}$ and when $\Delta_{k}^{n} \tau \geq t_{0}$, where $t_{0}=t_{0}(\varepsilon)$ is such that $\left|H_{\varphi}(t) / t-\breve{\beta}(\varphi)\right|<\varepsilon$ whenever $0<t<t_{0}$. We then take the limits when $n \rightarrow \infty$ and use the fact that $\varepsilon>0$ is arbitrary. 
It only remains to check that (4.2) implies (4.1) whenever $\bar{\delta}^{n} \rightarrow 0$. Indeed, by Jensen's inequality,

$$
\begin{aligned}
\mathrm{E}\left(\frac{1}{t_{n}^{n}} \sum_{k=1}^{n} \Delta_{k}^{n} \tau \mathbf{1}_{\left\{\Delta_{k}^{n} \tau \geq t_{0}\right\}}\right)^{2} & \leq \frac{1}{t_{0}^{\varepsilon}} \mathrm{E}\left(\frac{1}{t_{n}^{n}} \sum_{k=1}^{n}\left(\Delta_{k}^{n} \tau\right)^{1+\varepsilon / 2}\right)^{2} \\
& \leq \frac{1}{t_{0}^{\varepsilon}} \mathrm{E}\left(\frac{1}{t_{n}^{n}} \sum_{k=1}^{n}\left(t_{k}^{n}-t_{k-1}^{n}\right)^{\varepsilon} \int_{t_{k-1}^{n}}^{t_{k}^{n}}|r(u)|^{2+\varepsilon} \mathrm{d} u\right) \\
& \leq \frac{m_{2+\varepsilon}(g)}{t_{0}^{\varepsilon}}\left(\bar{\delta}^{n}\right)^{\varepsilon},
\end{aligned}
$$

which converges to 0 .

\section{Central limit theorems}

In this section we investigate conditions for the asymptotic normality of the estimators (1.5). In the case of a true Lévy process, Figueroa-López [13] proved this result assuming that $\varphi$ is bounded, $v$ continuous, and such that $\varphi(x)=o(|x|)$ as $x \rightarrow 0$. The random clock case is more challenging, as in this case $\hat{\beta}_{n}$ is not the sum of independent random variables. We use the central limit theorems for martingale differences (see, e.g. [3, Theorem 18.1]). Specifically, given a filtration $\left\{\mathcal{F}_{k}^{n}\right\}_{k \geq 0}$ for each $n \geq 0$, if $\xi_{k, n}$ is $\mathcal{F}_{k}^{n}$-measurable and $\mathrm{E}\left[\xi_{k, n} \mid \mathcal{F}_{k-1}^{n}\right]=0$, then

$$
S_{n}:=\sum_{k=1}^{\infty} \xi_{k, n} \stackrel{\mathrm{D}}{\rightarrow} \sigma \mathcal{N}(0,1)
$$

for a constant $\sigma \geq 0$, provided that the two conditions below are satisfied as $n \rightarrow \infty$ for any $\varepsilon>0$ :

$$
\sum_{k=1}^{\infty} \mathrm{E}\left[\xi_{k, n}^{2} \mid \mathcal{F}_{k-1}^{n}\right] \stackrel{\mathrm{P}}{\rightarrow} \sigma^{2}, \quad \sum_{k=1}^{\infty} \mathrm{E}\left[\xi_{k, n}^{2} \mathbf{1}_{\left|\xi_{k, n}\right| \geq \varepsilon}\right] \rightarrow 0 .
$$

In this section we take

$$
\mathcal{F}_{k}^{n}:=\sigma\left(X_{u}: u \leq t_{k}^{n}\right) \vee \sigma\left(\tau(u): u \leq t_{k}^{n}\right)
$$

for given sampling points $0=t_{0}^{n}<\cdots<t_{n}^{n}:=T_{n}$. We consider the following martingale difference sequence:

$$
\xi_{k, n}:=T_{n}^{-1 / 2}\left(\varphi\left(X_{t_{k}^{n}}-X_{t_{k-1}^{n}}\right)-\mathrm{E}\left[\varphi\left(X_{t_{k}^{n}}-X_{t_{k-1}^{n}}\right) \mid \mathcal{F}_{k-1}^{n}\right]\right)
$$

for $1 \leq k \leq n$, and $\xi_{k, n}=0$ otherwise. Define $\mathcal{F}_{t}^{Z}:=\sigma\left(Z_{u}: u \leq t\right)$ and $\mathcal{F}_{t}^{\tau}:=\sigma\left(\tau_{u}: u \leq t\right)$. Note that if $\varphi$ satisfies Proposition 3.1(i) and $\mathrm{E} \tau(t)<\infty$ for all $t \geq 0$, then $\mathrm{E}\left|H_{\varphi}\left(\Delta_{k}^{n} \tau\right)\right|<\infty$ and

$$
\mathrm{E}\left[\varphi\left(X_{t_{k}^{n}}-X_{t_{k-1}^{n}}\right) \mid \mathcal{F}_{k-1}^{n}\right]=\mathrm{E}\left[H_{\varphi}\left(\Delta_{k}^{n} \tau\right) \mid \mathcal{F}_{t_{k-1}}^{\tau}\right],
$$

where we recall that $\Delta_{k}^{n} \tau:=\tau\left(t_{k}^{n}\right)-\tau\left(t_{k-1}^{n}\right)$ and $H_{\varphi}(t):=\mathrm{E} \varphi\left(Z_{t}\right)$ (see the end of Appendix B for more details on (5.5)). We can then write (5.4) as

$$
\xi_{k, n}:=T_{n}^{-1 / 2}\left(\varphi\left(X_{t_{k}^{n}}-X_{t_{k-1}^{n}}\right)-\mathrm{E}\left[H_{\varphi}\left(\Delta_{k}^{n} \tau\right) \mid \mathcal{F}_{t_{k-1}}^{\tau}\right]\right)
$$


Also, we have

$$
S_{n}:=\sum_{k=1}^{\infty} \xi_{k, n}=T_{n}^{1 / 2}\left(\hat{\beta}_{n}(\varphi)-\check{\beta}_{n}(\varphi)\right)
$$

where

$$
\check{\beta}_{n}(\varphi):=\frac{1}{T_{n}} \sum_{k=1}^{n} \mathrm{E}\left[H_{\varphi}\left(\Delta_{k}^{n} \tau\right) \mid \mathcal{F}_{t_{k-1}}^{\tau}\right] .
$$

Our first task is to find conditions for

$$
\check{\beta}_{n}(\varphi) \stackrel{\mathrm{P}}{\rightarrow} \bar{\zeta}(g) \breve{\beta}(\varphi) \quad \text { as } n \rightarrow \infty .
$$

Lemma 5.1. Consider the model (1.3)-(1.4) under the setting of Proposition 3.1(a), (b), and (i). Also, assume that $g$ is Lipschitz on $\mathbb{R}$, satisfying (4.2) for some $\varepsilon>0$, and that $\tilde{r}$ satisfies (3.4) and Proposition 3.2(b'). Then, (5.7) holds whenever $T_{n} \nearrow \infty$ and $\bar{\delta}^{n} \rightarrow 0$.

Proof. Since $\mathrm{E} \check{\beta}_{n}(\varphi)=\mathrm{E} \beta_{n}(\varphi)$, it follows that $\lim _{n \rightarrow \infty} \mathrm{E} \check{\beta}_{n}(\varphi)=\bar{\zeta}(g) \breve{\beta}(\varphi)$ in light of Theorem 4.1. Hence, it suffices to show that

$$
\lim _{n \rightarrow \infty} \operatorname{var}\left(\check{\beta}_{n}(\varphi)\right)=0 .
$$

For a given $\varepsilon>0$, let $t_{0}:=t_{0}(\varepsilon)$ be such that $\left|H_{\varphi}(t) / t-\breve{\beta}(\varphi)\right|<\varepsilon$, whenever $0<t<t_{0}$. Next, decomposing $H_{\varphi}\left(\Delta_{k}^{n} \tau\right)$ as

$$
\left(\frac{1}{\Delta_{k}^{n} \tau} H_{\varphi}\left(\Delta_{k}^{n} \tau\right)-\breve{\beta}(\varphi)\right) \Delta_{k}^{n} \tau\left(\mathbf{1}_{\left\{\Delta_{k}^{n} \tau<t_{0}\right\}}+\mathbf{1}_{\left\{\Delta_{k}^{n} \tau \geq t_{0}\right\}}\right)+\breve{\beta}(\varphi) \Delta_{k}^{n} \tau,
$$

with the convention that $0 / 0=0$, and using (2.8), it follows that

$$
\operatorname{var}\left(\check{\beta}_{n}(\varphi)\right) \leq 4 \varepsilon^{2} \mathrm{E}\left[\rho_{n, 1}^{2}\right]+4\left(M_{\varphi}+|\breve{\beta}(\varphi)|\right) \mathrm{E}\left[\rho_{n, 2}^{2}\right]+4 \breve{\beta}(\varphi)^{2} \operatorname{var}\left(\rho_{n, 1}\right),
$$

where

$$
\rho_{n, 1}:=\frac{1}{T_{n}} \sum_{k=1}^{n} \mathrm{E}\left[\Delta_{k}^{n} \tau \mid \mathcal{F}_{t_{k-1}}^{\tau}\right], \quad \rho_{n, 2}:=\frac{1}{T_{n}} \sum_{k=1}^{n} \mathrm{E}\left[\Delta_{k}^{n} \tau \mathbf{1}_{\left\{\Delta_{k}^{n} \tau \geq t_{0}\right\}} \mid \mathcal{F}_{t_{k-1}}^{\tau}\right] .
$$

First, we note that (4.2) implies (3.1) and, by Jensen's inequality,

$$
\mathrm{E} \rho_{n, 1}^{2} \leq \frac{1}{T_{n}} \sum_{k=1}^{n} \int_{t_{k-1}}^{t_{k}} \operatorname{E} r^{2}(u) \mathrm{d} u \leq m_{2}(g) .
$$

Let $I_{k}^{n}:=\left[t_{k-1}^{n}, t_{k}^{n}\right]$ and $\delta_{k}^{n}:=t_{k}^{n}-t_{k-1}^{n}$. Following a procedure similar to (4.6), we obtain

$$
\rho_{n, 2}^{2} \leq \frac{t_{0}^{-\varepsilon}}{T_{n}} \sum\left(\delta_{k}^{n}\right)^{\varepsilon} \mathrm{E}\left[\int_{t_{k-1}^{n}}^{t_{k}^{n}}|\operatorname{rg}(\tilde{r}(u))|^{2+\varepsilon} \mathrm{d} u \mid \mathcal{F}_{t_{k-1}}^{\tau}\right]
$$

Then, using (4.2), $\mathrm{E} \rho_{n, 2}^{2} \leq t_{0}^{-\varepsilon}\left(\bar{\delta}^{n}\right)^{\varepsilon} m_{2+\varepsilon}(g) \rightarrow 0$. Let us analyze the last term in (5.9). First,

$$
\operatorname{var}\left(\rho_{n, 1}\right) \leq 2 \operatorname{var}\left(\rho_{n, 1}-\frac{1}{T_{n}} \tau\left(T_{n}\right)\right)+2 \operatorname{var}\left(\frac{1}{T_{n}} \tau\left(T_{n}\right)\right) .
$$


Using Jensen's inequality and the fact that

$$
\mathrm{E}\left(\mathrm{E}\left[r(u) \mid \mathcal{F}_{t_{k-1}^{n}}^{\tau}\right]-r(u)\right)^{2} \leq \mathrm{E}\left(r\left(t_{k-1}^{n}\right)-r(u)\right)^{2},
$$

the first term on the right-hand side of (5.10) can be bounded as follows:

$$
\frac{1}{T_{n}} \sum_{k=1}^{n} \int_{t_{k-1}^{n}}^{t_{k}^{n}} \mathrm{E}\left(r\left(t_{k-1}^{n}\right)-r(u)\right)^{2} \mathrm{~d} u \leq \frac{K}{T_{n}} \sum_{k=1}^{n} \delta_{k}^{n} \mathrm{E} \sup _{u \in I_{k}^{n}}\left|\tilde{r}(u)-\tilde{r}\left(t_{k-1}^{n}\right)\right|^{2},
$$

where $K$ is the Lipschitz constant of $g$. This converges to 0 in light of (3.7) and (3.4). The second term on the right-hand side of (5.10) converges to 0 since

$$
\operatorname{var}\left(\frac{1}{T_{n}} \tau\left(T_{n}\right)\right) \leq \mathrm{E}\left(\frac{1}{T_{n}} \int_{0}^{T_{n}} r(u) \mathrm{d} u-\bar{\zeta}(g)\right)^{2} \rightarrow 0 \quad \text { as } n \rightarrow \infty .
$$

The above limit is a consequence of the ergodic theorem (1.11) and the fact that

$$
\left(\frac{1}{T_{n}} \int_{0}^{T_{n}} r(u) \mathrm{d} u\right)^{2}
$$

is uniformly integrable, which in turn is guaranteed by (4.2). We finally conclude that

$$
\limsup _{n \rightarrow \infty} \operatorname{var}\left(\check{\beta}_{n}(\varphi)\right) \leq 4 m_{2}(g) \varepsilon^{2},
$$

and since $\varepsilon$ is arbitrary, (5.8) follows.

Proposition 5.1. Suppose that the conditions of Lemma 5.1 hold true and also that $\varphi^{2}$ satisfies Conditions 2.1 and (2.8). Then, when $T_{n} \nearrow \infty$ and $\bar{\delta}^{n} \rightarrow 0$,

$$
T_{n}^{1 / 2}\left(\hat{\beta}_{n}(\varphi)-\check{\beta}_{n}(\varphi)\right) \stackrel{\mathrm{D}}{\rightarrow} \sigma(\varphi) \mathcal{N}(0,1),
$$

with $\sigma^{2}(\varphi):=\bar{\zeta}(g) \breve{\beta}\left(\varphi^{2}\right)$

Proof. We need to check that (5.2) holds for (5.3) and (5.6). First,

$$
\sigma_{n}^{2}:=\sum_{k=1}^{\infty} \mathrm{E}\left[\xi_{k, n}^{2} \mid \mathcal{F}_{k-1}^{n}\right]=\check{\beta}_{n}\left(\varphi^{2}\right)-\frac{1}{T_{n}} \sum_{k=1}^{n}\left(\mathrm{E}\left[H_{\varphi}\left(\Delta_{k}^{n} \tau\right) \mid \mathcal{F}_{t_{k-1}}^{\tau}\right]\right)^{2} .
$$

In light of Lemma 5.1, $\check{\beta}_{n}\left(\varphi^{2}\right) \stackrel{\text { P }}{\rightarrow} \sigma^{2}(\varphi)$. The second term on the right-hand side, which we denote by $A_{n}$, converges in probability to 0 since

$$
\begin{aligned}
\mathrm{P}\left(\left|A_{n}\right| \geq \varepsilon\right) & \leq \frac{1}{\varepsilon T_{n}} \mathrm{E} \sum_{k=1}^{n}\left(\mathrm{E}\left[H_{\varphi}\left(\Delta_{k}^{n} \tau\right) \mid \mathcal{F}_{t_{k-1}}^{\tau}\right]\right)^{2} \\
& \leq \frac{M_{\varphi}^{2} m_{2}(g)}{\varepsilon} \frac{1}{T_{n}} \sum_{k=1}^{n}\left(t_{k}^{n}-t_{k-1}^{n}\right)^{2} \\
& \rightarrow 0 \quad \text { as } n \rightarrow \infty .
\end{aligned}
$$


Now consider

$$
B_{n}:=\frac{1}{T_{n}} \sum_{k=1}^{n} \mathrm{E}\left[\varphi^{2}\left(X_{t_{k}}-X_{t_{k-1}}\right) \mathbf{1}_{\left\{\left|\varphi\left(X_{t_{k}}-X_{t_{k-1}}\right)\right| \geq T_{n}^{1 / 2} \varepsilon / 2\right\}}\right] .
$$

Fix a $T_{0}>0$, and let $t_{0}>0$ be such that $\left|H_{\varphi^{2}} \mathbf{1}_{\left\{|\varphi| \geq T_{0}\right\}}(t)\right| \leq 2 t \beta\left(\varphi^{2} \mathbf{1}_{\left\{|\varphi| \geq T_{0}\right\}}\right)$ for all $0<t<t_{0}$. Then, conditioning on $\left\{\tau_{k}^{n}\right\}_{k=1}^{n}$, for large enough $n$,

$$
B_{n} \leq 2 \beta\left(\varphi^{2} \mathbf{1}_{\left\{|\varphi| \geq T_{0}\right\}}\right) \frac{1}{T_{n}} \sum_{k=1}^{n} \mathrm{E} \Delta_{k}^{n} \tau+M_{\varphi^{2}} \frac{1}{T_{n}} \sum_{k=1}^{n} \mathrm{E}\left[\Delta_{k}^{n} \tau \mathbf{1}_{\left\{\Delta_{k}^{n} \tau>t_{0}\right\}}\right]
$$

where limsup is bounded by $2 \beta\left(\varphi^{2} \mathbf{1}_{\left\{|\varphi| \geq T_{0}\right\}}\right)$ since $\left(1 / T_{n}\right) \sum_{k=1}^{n} \mathrm{E} \Delta_{k}^{n} \tau \leq m_{2}^{1 / 2}$ and (4.2) implies (4.1) (see the last part in the proof of Theorem 4.1), which in turn implies that the second term on the right-hand side above vanishes. Since $T_{0}$ can be made arbitrarily large, $\lim \sup _{n \rightarrow \infty} B_{n}=0$. Next, conditioning on $\mathcal{F}_{k-1}^{n}$,

$$
\begin{aligned}
C_{n} & :=\frac{1}{T_{n}} \sum_{k=1}^{n} \mathrm{E}\left[\varphi^{2}\left(X_{t_{k}}-X_{t_{k-1}}\right) \mathbf{1}_{\left\{\left|\mathrm{E}\left[H_{\varphi}\left(\Delta_{k}^{n} \tau\right) \mid \mathcal{F}_{t_{k-1}}^{\tau}\right]\right| \geq T_{n}^{1 / 2} \varepsilon / 2\right\}}\right] \\
& \leq M_{\varphi^{2}} \frac{1}{T_{n}} \sum_{k=1}^{n} \mathrm{E}\left[\mathrm{E}\left[\Delta_{k}^{n} \tau \mid \mathcal{F}_{t_{k-1}}^{\tau}\right] \mathbf{1}_{\left\{\left|\mathrm{E}\left[\Delta_{k}^{n} \tau \mid \mathcal{F}_{t_{k-1}}^{\tau}\right]\right| \geq M_{\varphi}^{-1} T_{n}^{1 / 2} \varepsilon / 2\right\}}\right], \\
& \leq M_{\varphi^{2}} M_{\varphi} \varepsilon^{-1} \frac{1}{T_{n}^{3 / 2}} \sum_{k=1}^{n} \mathrm{E}\left[\mathrm{E}\left[\Delta_{k}^{n} \tau \mid \mathcal{F}_{t_{k-1}}^{\tau}\right]^{2}\right],
\end{aligned}
$$

which can be shown to converge to 0 as $D_{n}$ below converges to 0 . Using Jensen's inequality,

$$
D_{n}:=\frac{1}{T_{n}} \sum_{k=1}^{n} \mathrm{E}\left[\mathrm{E}\left[H_{\varphi}\left(\Delta_{k}^{n} \tau\right) \mid \mathcal{F}_{t_{k-1}}^{\tau}\right]^{2}\right] \leq \frac{M_{\varphi}^{2}}{T_{n}} \sum_{k=1}^{n}\left(t_{k}^{n}-t_{k-1}^{n}\right) \int_{t_{k-1}^{n}}^{t_{k}^{n}} \mathrm{E} r^{2}(u) \mathrm{d} u,
$$

which clearly converges to 0 in light of (4.2). Thus, we obtain the second limit in (5.2) because

$$
\sum_{k=0}^{\infty} \mathrm{E}\left[\xi_{k, n}^{2} \mathbf{1}_{\left\{\left|\xi_{k, n}\right| \geq \varepsilon\right\}}\right] \leq 2 B_{n}+2 C_{n}+D_{n}
$$

In light of the central limit theorem for martingale differences stated at the beginning of this section, we obtain (5.11).

We proceed to show a central limit theorem for $\check{\beta}_{n}(\varphi)$ of the form

$$
T_{n}^{1 / 2}\left(\check{\beta}_{n}(\varphi)-\bar{\zeta}(g) \breve{\beta}(\varphi)\right) \stackrel{\mathrm{D}}{\rightarrow} \breve{\beta}(\varphi) \Gamma^{1 / 2}(g) \mathcal{N}(0,1)
$$

for a certain positive constant $\Gamma(g)$. This result suggests a central limit theorem of the form

$$
T_{n}^{1 / 2}\left(\hat{\beta}_{n}(\varphi)-\bar{\zeta}(g) \breve{\beta}(\varphi)\right) \stackrel{\mathrm{D}}{\rightarrow}\left(\sigma^{2}(\varphi)+\breve{\beta}(\varphi)^{2} \Gamma(g)\right)^{1 / 2} \mathcal{N}(0,1) .
$$

However, we have not been able to obtain such a result and we expect to address this issue in a future work. We shall need an additional assumption on the rate of convergence in (2.6). 
Condition 5.1. There exists a $t_{0}>0$ such that

$$
\left|\frac{1}{t} \mathrm{E} \varphi\left(X_{t}\right)-\breve{\beta}(\varphi)\right| \leq k_{0} t
$$

for any $0<t<t_{0}$ and a constant $k_{0}$ independent of $t$.

Remark 5.1. Condition 5.1 turns out to hold for a wide class of functions $\varphi$, such as the following:

1. $\varphi$ is supported on an interval $[c, d] \subset \mathbb{R} \backslash\{0\}$, where $\varphi$ is continuous with continuous derivative (cf. [11]);

2. $\varphi \in C^{2}$ vanishes in a neighborhood of the origin and, for each $i=0,1,2,\left|\varphi^{(i)}\right|$ is bounded by an element $g_{i}$ in the class $\delta(v)$ of (2.5) (cf. [12]).

Proposition 5.2. Suppose that Condition 5.1 holds as well as the conditions of Proposition 5.1. Then, (5.12) holds true provided that $T_{n} \rightarrow \infty$ and $T_{n} \bar{\delta}^{n} \rightarrow 0$ as $n \rightarrow \infty$.

Proof. We recall that, under the stated conditions, the diffusion $\{\tilde{r}(t)\}_{t \geq 0}$ obeys the central limit theorem

$$
\sqrt{t}\left(\frac{1}{t} \int_{0}^{t} g(\tilde{r}(u)) \mathrm{d} u-\int g(x) \zeta(\mathrm{d} x)\right) \stackrel{\mathrm{D}}{\rightarrow} \mathcal{N}(0, \Gamma(g))
$$

for a certain constant $\Gamma(g) \geq 0$ (see [22] for an explicit formula for $\Gamma(g)$ and the references therein for a proof). Also,

$$
T_{n}^{1 / 2}\left(\check{\beta}_{n}(\varphi)-\bar{\zeta}(g) \breve{\beta}(\varphi)\right)=\breve{\beta}(\varphi) T_{n}^{-1 / 2} \int_{0}^{T_{n}}(g(\tilde{r}(u))-\bar{\zeta}(g)) \mathrm{d} u+R_{n},
$$

where

$$
\begin{aligned}
R_{n}:= & T_{n}^{-1 / 2} \sum_{k=1}^{n} \mathrm{E}\left[\left(\frac{1}{\Delta_{k}^{n}} H_{\varphi}\left(\Delta_{k}^{n} \tau\right)-\breve{\beta}(\varphi)\right) \Delta_{k}^{n} \tau \mid \mathcal{F}_{t_{k-1}}^{\tau}\right] \\
& +\breve{\beta}(\varphi) T_{n}^{1 / 2}\left(\frac{1}{T_{n}} \sum_{k=1}^{n} \mathrm{E}\left[\Delta_{k}^{n} \tau \mid \mathcal{F}_{t_{k-1}}^{\tau}\right]-\frac{1}{T_{n}} \int_{0}^{T_{n}} r(u) \mathrm{d} u\right)
\end{aligned}
$$

Thus, to show (5.12) it suffices that $R_{n}$ converges to 0 in probability. Denote by $A_{n}$ the term on the right-hand side of (5.16). Note that without loss of generality we can assume that (5.14) holds for all $t>0$. Then,

$$
\begin{aligned}
\mathrm{P}\left(\left|A_{n}\right| \geq \varepsilon\right) & \leq \frac{k_{0}}{\varepsilon} T_{n}^{-1 / 2} \sum_{k=1}^{n} \mathrm{E}\left(\Delta_{k}^{n} \tau\right)^{2} \\
& \leq \frac{k_{0}}{\varepsilon} T_{n}^{-1 / 2} \sum_{k=1}^{n}\left(t_{k}^{n}-t_{k-1}^{n}\right) \mathrm{E} \int_{t_{k-1}^{n}}^{t_{k}^{n}} r^{2}(u) \mathrm{d} u \\
& \leq \frac{m_{2}(g) k_{0}}{\varepsilon} T_{n}^{1 / 2} \bar{\delta}^{n},
\end{aligned}
$$

which converges to 0 . For (5.17), we proceed as before when proving that the first term on the right-hand side of (5.10) converges to 0 . Indeed, denoting by $B_{n}$ the term on the left-hand 
side of (5.17) and using Markov's inequality, the Lipschitz condition of $g$, (3.4), and (3.7), we obtain

$$
\mathrm{P}\left(\left|B_{n}\right| \geq \varepsilon\right) \leq \frac{1}{\varepsilon^{2}} \sum_{k=1}^{n} \delta_{k}^{n} \mathrm{E} \sup _{u \in I_{k}^{n}}\left|r(u)-r\left(t_{k-1}^{n}\right)\right|^{2} \leq \frac{K}{\varepsilon^{2}} T_{n} \bar{\delta}^{n} \rightarrow 0 \quad \text { as } n \rightarrow \infty .
$$

This concludes the proof.

Note that $T_{n}^{1 / 2}\left(\hat{\beta}_{n}(\varphi)-\bar{\zeta}(g) \breve{\beta}(\varphi)\right)$ can be decomposed as follows:

$$
T_{n}^{1 / 2}\left(\hat{\beta}_{n}(\varphi)-\breve{\beta}_{n}(\varphi)\right)+\breve{\beta}(\varphi) T_{n}^{-1 / 2} \int_{0}^{T_{n}}(g(\tilde{r}(u))-\bar{\zeta}(g)) \mathrm{d} u+R_{n},
$$

where $R_{n}$ is defined as in (5.16). In the proof of Proposition 5.2, it was shown that $R_{n}$ converges to 0 in probability, while each of the first two terms converge to a normal distribution in light of (5.11) and (5.15). To conclude (5.13), it will suffice to show that the first two terms converge jointly in distribution, an issue that we are currently pursuing.

\section{Appendix A. A moment estimate for diffusions}

In this appendix we prove the moment estimate (3.7) for the solution $\tilde{r}$ of the stochastic differential equation (3.5) under the linear growth condition (3.6). The ideas are classical (see, e.g. the solution to Problem 5.3.15 of [18]). Below, $k_{m}$ stands for a generic constant depending on $m$. First, note that

$$
\left|\tilde{r}\left(s_{0}+s\right)-\tilde{r}\left(s_{0}\right)\right|^{2 m} \leq k_{m}\left(\left|\int_{s_{0}}^{s_{0}+s} b(\tilde{r}(u)) \mathrm{d} u\right|^{2 m}+\left|\int_{s_{0}}^{s_{0}+s} \sigma(\tilde{r}(u)) \mathrm{d} W_{u}\right|^{2 m}\right) .
$$

By Jensen's inequality and (3.6), $\left|\int_{s_{0}}^{s_{0}+s} b(\tilde{r}(u)) \mathrm{d} u\right|^{2 m}$ can be bounded as follows:

$$
\begin{aligned}
s^{2 m-1} & \int_{s_{0}}^{s_{0}+s}|b(\tilde{r}(u))|^{2 m} \mathrm{~d} u \\
& \leq k_{m} s^{2 m-1} \int_{s_{0}}^{s_{0}+s}\left(1+|\tilde{r}(u)|^{2 m}\right) \mathrm{d} u \\
& \leq k_{m} s^{2 m-1}\left(s+s\left|\tilde{r}\left(s_{0}\right)\right|^{2 m}+\int_{s_{0}}^{s_{0}+s}\left|\tilde{r}(u)-\tilde{r}\left(s_{0}\right)\right|^{2 m} \mathrm{~d} u\right) .
\end{aligned}
$$

Let $\tau_{k}:=\inf \left\{s \geq 0:\left|\tilde{r}\left(s+s_{0}\right)\right| \geq k\right\}$. By the Davis-Burkhölder-Gundy inequality,

$$
\begin{aligned}
\mathrm{E} \sup _{s \leq h \wedge \tau_{k}}\left|\int_{s_{0}}^{s_{0}+s} \sigma(\tilde{r}(u)) \mathrm{d} W_{u}\right|^{2 m} & \leq k_{m} \mathrm{E}\left|\int_{s_{0}}^{s_{0}+h \wedge \tau_{k}} \sigma^{2}(\tilde{r}(u)) \mathrm{d} u\right|^{m} \\
& \leq k_{m} h^{m-1} \mathrm{E} \int_{s_{0}}^{s_{0}+h \wedge \tau_{k}} \sigma^{2 m}(\tilde{r}(u)) \mathrm{d} u
\end{aligned}
$$

As with $b(\cdot)$, we have the following bound for any $s \geq 0$ :

$$
\int_{s_{0}}^{s_{0}+s} \sigma^{2 m}(\tilde{r}(u)) \mathrm{d} u \leq k_{m}\left(s+s\left|\tilde{r}\left(s_{0}\right)\right|^{2 m}+\int_{s_{0}}^{s_{0}+s}\left|\tilde{r}(u)-\tilde{r}\left(s_{0}\right)\right|^{2 m} \mathrm{~d} u\right) .
$$


Then, using $0<h \leq 1, \operatorname{Esup}_{s \leq h \wedge \tau_{k}}\left|\tilde{r}\left(s_{0}+s\right)-\tilde{r}\left(s_{0}\right)\right|^{2 m}$ can be upper bounded by

$$
\begin{aligned}
k_{m} \mathrm{E} & \sup _{s \leq h \wedge \tau_{k}}\left|\int_{s_{0}}^{s_{0}+s} b(\tilde{r}(u)) \mathrm{d} u\right|^{2 m}+k_{m} \mathrm{E} \sup _{s \leq h \wedge \tau_{k}}\left|\int_{s_{0}}^{s_{0}+s} \sigma(\tilde{r}(u)) \mathrm{d} W_{u}\right|^{2 m} \\
& \leq k_{m} h^{m}+k_{m} h^{m} \mathrm{E}\left|\tilde{r}\left(s_{0}\right)\right|^{2 m}+k_{m} h^{m-1} \int_{0}^{h} \mathrm{E} \sup _{s \leq u \wedge \tau_{k}}\left|\tilde{r}\left(s_{0}+s\right)-\tilde{r}\left(s_{0}\right)\right|^{2 m} \mathrm{~d} u .
\end{aligned}
$$

Defining $\gamma_{k}(h):=\operatorname{Esup}_{s \leq h \wedge \tau_{k}}\left|\tilde{r}\left(s_{0}+s\right)-\tilde{r}\left(s_{0}\right)\right|^{2 m}$, we obtain the inequality

$$
\gamma_{k}(h) \leq k_{m} h^{m}\left(1+\mathrm{E}\left|\tilde{r}\left(s_{0}\right)\right|^{2 m}\right)+k_{m} \int_{0}^{h} \gamma_{k}(u) \mathrm{d} u .
$$

Finally, by Gronwall's inequality (see [18]), $\gamma_{k}(h) \leq k_{m} h^{m}\left(1+\mathrm{E}\left|\tilde{r}\left(s_{0}\right)\right|^{2 m}\right) \mathrm{e}^{k_{m} h}$. Inequality (3.7) will follow by letting $k \rightarrow \infty$.

\section{Appendix B. Conditional expectation given the random clock}

On several occasions we used conditional expectations of the time-changed Lévy model $X_{t}:=Z_{\tau(t)}$ given the random clock $\tau$ and/or past evolution of $X$. In this appendix we intend to formalize this procedure under the assumption that $Z$ and $\tau$ are independent.

(i) Let $0 \leq t_{0}<\cdots<t_{n}<\infty$, and let $\tau_{k}:=\tau\left(t_{k}\right)$. For given $0 \leq s_{0} \leq \cdots \leq s_{n}<\infty$, we first show that the distribution of $Z_{\tau_{1}}-Z_{\tau_{0}}, \ldots, Z_{\tau_{n}}-Z_{\tau_{n-1}}$ given $\tau_{0}=s_{0}, \ldots, \tau_{n}=s_{n}$ is the same as that of $Z_{s_{1}}-Z_{s_{0}}, \ldots, Z_{s_{n}}-Z_{s_{n-1}}$. Let

$$
M_{s_{0}, \ldots, s_{n}}\left(u_{1}, \ldots, u_{n}\right)=\mathrm{E} \prod_{k=1}^{n} \exp \left(\mathrm{i} u_{k}\left(Z_{s_{k}}-Z_{s_{k-1}}\right)\right)
$$

let $A \in \sigma\left(\tau_{0}, \ldots, \tau_{n}\right)$, and let $\kappa_{m}(t)=\sum_{j=1}^{m^{2}}(j / m) \mathbf{1}_{[(j-1) / m, j / m)}(t)+m \mathbf{1}_{[m, \infty)}(t)$. First, by the right continuity of $Z$ and the dominated convergence theorem,

$$
\mathrm{E} \prod_{k=1}^{n} \exp \left(\mathrm{i} u_{k}\left(Z_{\tau_{k}}-Z_{\tau_{k-1}}\right)\right) \chi_{A}=\lim _{m \rightarrow \infty} \mathrm{E} \prod_{k=1}^{n} \exp \left(\mathrm{i} u_{k}\left(Z_{\kappa_{m}\left(\tau_{k}\right)}-Z_{\kappa_{m}\left(\tau_{k-1}\right)}\right)\right) \chi_{A} .
$$

Using the independence of $Z$ and $\tau$, the expectation after the limit in the previous equation can be expressed as follows:

$$
\begin{aligned}
& \sum_{1 \leq j_{0} \leq \cdots \leq j_{n} \leq m^{2}} \mathrm{E} \prod_{k=1}^{n} \exp \left(\mathrm{i} u_{k}\left(Z_{j_{k} / m}-Z_{j_{k-1} / m}\right)\right) \prod_{k=0}^{n} \mathbf{1}_{\left[\left(j_{k}-1\right) / m, j_{k} / m\right)}\left(\tau_{k}\right) \chi_{A} \\
& =\sum_{j_{0} \leq \cdots \leq j_{n}} M_{j_{0} / m, \ldots, j_{n} / m}\left(u_{1}, \ldots, u_{n}\right) \mathrm{E} \prod_{k=0}^{n} \mathbf{1}_{\left[\left(j_{k}-1\right) / m, j_{k} / m\right)}\left(\tau_{k}\right) \chi_{A} \\
& =\mathrm{E} M_{\kappa_{m}\left(\tau_{0}\right), \ldots, \kappa_{m}\left(\tau_{n}\right)}\left(u_{1}, \ldots, u_{n}\right) \chi_{A} .
\end{aligned}
$$

Using dominated convergence and right continuity of $Z$, the last expression converges to E $M_{\tau_{0}, \ldots, \tau_{n}}\left(u_{1}, \ldots, u_{n}\right) \chi_{A}$ as $m \rightarrow \infty$. Thus, we can conclude that

$$
\mathrm{E}\left[\prod_{k=1}^{n} \exp \left(\mathrm{i} u_{k}\left(Z_{\tau_{k}}-Z_{\tau_{k-1}}\right)\right) \mid \tau_{0}, \ldots, \tau_{n}\right]=M_{\tau_{0}, \ldots, \tau_{n}}\left(u_{1}, \ldots, u_{n}\right) .
$$


We could similarly show that the distribution of $Z_{\tau_{1}}-Z_{\tau_{0}}, \ldots, Z_{\tau_{n}}-Z_{\tau_{n-1}}$ given

$$
\mathcal{F}^{\tau}:=\sigma(\tau(t): t \geq 0)
$$

is the same distribution as that of $Z_{s_{1}}-Z_{s_{0}}, \ldots, Z_{s_{n}}-Z_{s_{n-1}}$ at $s_{0}=\tau_{0}, \ldots, s_{n}=\tau_{n}$.

(ii) As a consequence of the previous result, if $g: \mathbb{R}^{n} \rightarrow \mathbb{R}_{+}$is such that

$$
\operatorname{Eg}\left(Z_{\tau_{1}}-Z_{\tau_{0}}, \ldots, Z_{\tau_{n}}-Z_{\tau_{n-1}}\right)<\infty
$$

then

$$
\operatorname{Eg}\left(Z_{\tau_{1}}-Z_{\tau_{0}}, \ldots, Z_{\tau_{n}}-Z_{\tau_{n-1}}\right)=\mathrm{E} G\left(\tau_{0}, \ldots, \tau_{n}\right),
$$

where $G\left(s_{0}, \ldots, s_{n}\right):=\mathrm{E} g\left(Z_{s_{1}}-Z_{s_{0}}, \ldots, Z_{s_{n}}-Z_{s_{n-1}}\right)$. Furthermore, if $g: \mathbb{R}^{n} \rightarrow \mathbb{R}_{+}$is continuous and there exists an $M<\infty$ such that $G\left(s_{0}, \ldots, s_{n}\right) \leq M$ whenever $0 \leq s_{0} \leq \cdots \leq s_{n}$, then (B.1) holds and, hence, (B.2) holds too. Indeed, by Fatou's lemma,

$$
\begin{aligned}
\operatorname{Eg} g & \left(Z_{\tau_{1}}-Z_{\tau_{0}}, \ldots, Z_{\tau_{n}}-Z_{\tau_{n-1}}\right) \\
& \leq \liminf _{m \rightarrow \infty} \mathrm{E} g\left(Z_{\kappa_{m}\left(\tau_{1}\right)}-Z_{\kappa_{m}\left(\tau_{0}\right)}, \ldots, Z_{\kappa_{m}\left(\tau_{n}\right)}-Z_{\kappa_{m}\left(\tau_{n-1}\right)}\right) \\
& \leq \liminf _{m \rightarrow \infty} \mathrm{E} G\left(\kappa_{m}\left(\tau_{0}\right), \ldots, \kappa_{m}\left(\tau_{n}\right)\right) \\
& \leq M .
\end{aligned}
$$

The above reasoning was used, for instance, to show (2.10) since, under assumption (2.8), $G\left(s_{0}, \ldots, s_{n}\right):=\left(1 / s_{n}\right) \sum_{k=1}^{n} H_{|\varphi|}\left(s_{k}-s_{k-1}\right) \leq M_{\varphi}$.

(iii) Let us show identity (5.5). Let $\varphi_{k}, \psi_{k}: \mathbb{R} \rightarrow \mathbb{R}_{+}, k=1, \ldots, n$, be continuous bounded functions, and let $0=t_{0} \leq t_{1}<\cdots<t_{n} \leq t<u<\infty$. Again, we write $\tau_{k}:=\tau\left(t_{k}\right)$. Then, conditioning on $\mathcal{F}^{\tau}$,

$$
\mathrm{E}\left[\varphi\left(Z_{\tau(u)}-Z_{\tau(t)}\right) \prod_{k=1}^{n} \varphi_{k}\left(Z_{\tau_{k}}\right) \psi_{k}\left(\tau_{k}\right)\right]=\mathrm{E}\left[H_{\varphi}(\tau(u)-\tau(t)) m\left(\tau_{1}, \ldots, \tau_{n}\right)\right],
$$

where $H_{\varphi}(t):=\mathrm{E} \varphi\left(Z_{t}\right)$, and $m\left(s_{1}, \ldots, s_{n}\right)=\mathrm{E} \prod_{k=1}^{n} \varphi_{k}\left(Z_{s_{k}}\right) \psi_{k}\left(s_{k}\right)$. Since

$$
m\left(\tau_{1}, \ldots, \tau_{n}\right)=\mathrm{E}\left[\prod_{k=1}^{n} \varphi_{k}\left(Z_{\tau_{k}}\right) \psi_{k}\left(\tau_{k}\right) \mid \mathcal{F}_{t}^{\tau}\right]
$$

the right-hand side of (B.3) can be written as follows:

$$
\mathrm{E}\left[\mathrm{E}\left[H_{\varphi}(\tau(u)-\tau(t)) \mid \mathcal{F}_{t}^{\tau}\right] \prod_{k=1}^{n} \varphi_{k}\left(Z_{\tau_{k}}\right) \psi_{k}\left(\tau_{k}\right)\right]
$$

Since $\mathcal{F}_{t}^{\tau} \subset \mathcal{F}_{t}^{X} \vee \mathcal{F}_{t}^{\tau}$, we conclude that

$$
\mathrm{E}\left[\varphi\left(X_{u}-X_{t}\right) \mid \mathcal{F}_{t}^{X} \vee \mathcal{F}_{t}^{\tau}\right]=\mathrm{E}\left[H_{\varphi}(\tau(u)-\tau(t)) \mid \mathcal{F}_{t}^{\tau}\right]
$$

\section{Acknowledgements}

The author would like to thank an anonymous referee for valuable comments and suggestions. Research partially supported by a grant from the US National Science Foundation (DMS0906919) and a 2008 Summer Faculty Grant from the Purdue Research Foundation. 


\section{References}

[1] Barndorff-Nielsen, O. E. (1998). Processes of normal inverse Gaussian type. Finance Stoch. 2, 41-68.

[2] Barndorff-Nielsen, O. E. And Shephard, N. (2001). Modelling by Lévy processess for financial economics. In Lévy Processes, Birkhäuser, Boston, MA, pp. 283-318.

[3] Billingsley, P. (1999). Convergence of Probability Measures. John Wiley, New York.

[4] Carr, P. And Wu, L. (2004). Time-changed Levy processes and option pricing. J. Financial Economics 71, $113-141$.

[5] Carr, P., Geman, H., Madan, D. and Yor, M. (2002). The fine structure of asset returns: an empirical investigation. J. Business 75, 305-332.

[6] Carr, P., Geman, H., Madan, D. and Yor, M. (2003). Stochastic volatility for Lévy processes. Math. Finance 13, 345-382.

[7] Chung, K. L. (2001). A Course in Probability Theory, 3rd edn. Academic Press, San Diego, CA.

[8] Eberlein, E. (2001). Application of generalized hyperbolic Lévy motions to finance. In Lévy Processes, Birkhäuser, Boston, MA, pp. 319-336.

[9] Eberlein, E. ANd Keller, U. (1995). Hyperbolic distribution in finance. Bernoulli 1, 281-299.

[10] Figueroa-LóPez, J. E. (2004). Nonparametric estimation of Lévy processes with a view towards mathematical finance. Doctoral Thesis, Georgia Institute of Technology.

[11] Figueroa-LóPez, J. E. (2008). Sieve-based confidence intervals and bands for Lévy densities. Preprint. Available at www.stat.purdue.edu/ figueroa.

[12] Figueroa-López, J. E. (2008). Small-time moment asymptotics for Lévy processes. Statist. Prob. Lett. 78, $3355-3365$.

[13] FigueroA-López, J. E. (2009). Nonparametric estimation for Lévy models based on discrete-sampling. In Optimality: The Third Erich L. Lehmann Symposium (IMS Lecture Notes Monogr. Ser. 57), Institute of Mathematical Statistics, Beachwood, Ohio, pp. 117-146.

[14] Figueroa-López, J. E. (2009). Nonparametric estimation of time-changed Lévy models under high-frequency data. Tech. Rep. Department of Statistics, Purdue University. Available at www.stat.purdue.edu/ figueroa.

[15] Figueroa-López, J. E. ANd Houdré, C. (2006). Risk bounds for the non-parametric estimation of Lévy processes. In High Dimensional Probability (IMS Lecture Notes Monogr. Ser. 51), Beachwood, Ohio, pp. 96116.

[16] JACOD, J. (2007). Asymptotic properties of power variations of Lévy processes. ESAIM Prob. Statist. 11, $173-196$.

[17] Kallenberg, O. (1997). Foundations of Modern Probability. Springer, New York.

[18] Karatzas, I. and Shreve, S. E. (1988). Brownian Motion and Stochastic Calculus. Springer, New York.

[19] Madan, D. B., CARR, P. ANd Chang, E. C. (1998). The variance gamma process and option pricing. Europ. Finance Rev. 2, 79-105.

[20] Mancini, C. (2009). Non-parametric threshold estimation for models with stochastic diffusion coefficient and jumps. Scand. J. Statist. 36, 270-296.

[21] Sato, K. I. (1999). Lévy Processes and Infinitely Divisible Distributions. Cambridge University Press.

[22] Van der Vaart, A. and van Zanten, H. (2005). Donsker theorems for diffusions: necessary and sufficient conditions. Ann. Prob. 33, 1422-1451.

[23] VAN ZANTEN, J. H. (2003). On uniform laws of large numbers for ergodic diffusions and consistency of estimators. Statist. Infer. Stoch. Process. 6, 199-213.

[24] Woerner, J. H. C. (2003). Variational sums and power variation: a unifying approach to model selection and estimation in semimartingale models. Statist. Decisions 21, 47-68.

[25] Woerner, J. H. C. (2007). Inference in Lévy-type stochastic volatility models. Adv. Appl. Prob. 39, 531-549. 